\title{
The Symplectic Topology of Projective Manifolds with Small Dual
}

\author{
Paul Biran and Yochay Jerby \\ Departement Mathematik, ETH, 8092 Zürich, Switzerland \\ Correspondence to be sent to: biran@math.ethz.ch
}

We study smooth projective varieties with small dual variety using methods from symplectic topology. For such varieties, we prove that the hyperplane class is an invertible element in the quantum cohomology of their hyperplane sections. We also prove that the affine part of such varieties are subcritical. We derive several topological and algebraic geometric consequences from that. The main tool in our work is the Seidel representation associated to Hamiltonian fibrations.

\section{Introduction and Summary of the Main Results}

In this paper, we study a special class of complex algebraic manifolds called projective manifolds with small dual. A projectively embedded algebraic manifold $X \subset \mathbb{C} P^{N}$ is said to have small dual if the dual variety $X^{*} \subset\left(\mathbb{C} P^{N}\right)^{*}$ has (complex) codimension $\geq 2$. Recall that the dual variety $X^{*}$ of a projectively embedded algebraic manifold $X \subset \mathbb{C} P^{N}$ is by definition the space of all hyperplanes $H \subset \mathbb{C} P^{N}$ that are not transverse to $X$, that is,

$$
X^{*}=\left\{H \in\left(\mathbb{C} P^{N}\right)^{*} \mid H \text { is somewhere tangent to } X\right\}
$$

Let us mention that for "most" manifolds the codimension of $X^{*}$ is 1 , however, in special situations the codimension might be larger. To measure to which extent $X$ deviates from

Received July 14, 2011; Revised June 29, 2012; Accepted July 2, 2012

Communicated by Prof. Yasha Eliashberg

(C) The Author(s) 2012. Published by Oxford University Press. All rights reserved. For permissions, please e-mail: journals.permissions@oup.com. 
the typical case one defines the defect of an algebraic manifold $X \subset \mathbb{C} P^{N}$ by

$$
\operatorname{def}(X)=\operatorname{codim}_{\mathbb{C}}\left(X^{*}\right)-1
$$

(Some authors call this quantity dual defect to distinguish it from other "defects" appearing in projective geometry, such as secant defect, see, for example, [32, 48]. In this paper, we will, however, stick to the wording "defect", which is attributed to A. Landman in $[20,21]$.)

Thus, we will call manifolds with small dual also manifolds with positive defect. Note that this is not an intrinsic property of $X$, but rather of a given projective embedding of $X$.

The class of algebraic manifolds with small dual was studied by many authors, for instance, see [5, 20, 21, 27, 31,45], see also [47] for a survey. The study of the relation between $X^{*}$ and the topology of $X$ (and its hyperplane sections) had been initiated earlier in [2]. These works show that manifolds with small dual have very special geometry. In this paper, we will show that such manifolds also exhibit unique properties from the point of view of symplectic topology.

Our main results are concerned with geometric properties of a smooth hyperplane section $\Sigma \subset X$ of a manifold $X \subset \mathbb{C} P^{N}$ with small dual, under the additional assumption that $b_{2}(X)=1$. (Here and in what follows, we denote by $b_{j}(X)=\operatorname{dim} H^{j}(X ; \mathbb{R})$ the $j$ 'th Betti-number of $X$.) By a well-known result of Ein [21], the assumption $b_{2}(X)=1$ implies that both $X$ and $\Sigma$ are Fano manifolds.

For a space $Y$, we will denote from now on by

$$
H^{*}(Y):=H^{*}(Y ; \mathbb{Z}) / \text { torsion }
$$

the torsion-free part of the integral cohomology $H^{*}(Y ; \mathbb{Z})$. Denote by $\mathrm{OH}^{*}(\Sigma ; \Lambda)=$ $\left(H^{\bullet}(\Sigma) \otimes \Lambda\right)^{*}$ the quantum cohomology ring of $\Sigma$ with coefficients in the Novikov ring $\Lambda=\mathbb{Z}\left[q, q^{-1}\right]$ (see below for our grading conventions), and endowed with the quantum product $*$. We prove the following theorem.

Theorem A. Let $X \subset \mathbb{C} P^{N}$ be an algebraic manifold with small dual, $b_{2}(X)=1$ and $\operatorname{dim}_{\mathbb{C}}(X) \geq 2$. Let $\Sigma$ be a smooth hyperplane section of $X$. Let $\omega$ be the restriction of the Fubini-Study Kähler form of $\mathbb{C} P^{N}$ to $\Sigma$. Then

$$
[\omega] \in \mathrm{OH}^{2}(\Sigma ; \Lambda)
$$

is an invertible element with respect to the quantum product. 
We will actually prove a slightly stronger result in Section 4 (see Theorem 4.2 and the discussion after it). In Theorem 9.1 in Section 9, we will establish a much more general, though less precise, version of this theorem.

A classical result of Lanteri and Struppa [33] (see also [2]) on the topology of projective manifolds with positive defect states that if $X \subset \mathbb{C} P^{N}$ is a projective manifold with $\operatorname{dim}_{\mathbb{C}} X=n$ and $\operatorname{def}(X)=k>0$, then

$$
b_{j}(X)=b_{j+2}(X) \forall n-(k-1) \leq j \leq n+k-1 .
$$

(In Section 6, we will reprove this fact using Morse theory). As we will see in Corollary $\mathrm{B}$, Theorem A implies stronger topological restrictions in the case $b_{2}(X)=1$.

As mentioned above, under the assumption $b_{2}(X)=1$ the manifold $\Sigma$ is Fano. The quantum cohomology $\mathrm{OH}^{*}(\Sigma ; \Lambda)=\left(H^{\bullet}(\Sigma) \otimes \Lambda\right)^{*}$ admits a grading induced from both factors $H^{\bullet}(\Sigma)$ and $\Lambda$. Here we grade $\Lambda$ by taking $\operatorname{deg}(q)=2 C_{\Sigma}$, where

$$
C_{\Sigma}=\min \left\{C_{1}^{\Sigma}(A)>0 \mid A \in \text { image }\left(\pi_{2}(\Sigma) \rightarrow H_{2}(\Sigma ; \mathbb{Z})\right)\right\} \in \mathbb{N}
$$

is the minimal Chern number of $\Sigma$. Here, we have denoted by $c_{1}^{\Sigma} \in H^{2}(\Sigma ; \mathbb{Z})$ the first Chern class of the tangent bundle $T \Sigma$ of $\Sigma$. Theorem A implies that the map

$$
*[\omega]: \mathrm{OH}^{*}(\Sigma ; \Lambda) \longrightarrow \mathrm{OH}^{*+2}(\Sigma ; \Lambda), \quad a \longmapsto a *[\omega]
$$

is an isomorphism. In our case, a computation of Ein [21] gives

$$
2 C_{X}=n+k+2, \quad 2 C_{\Sigma}=n+k .
$$

(It is well known, by a result of Landman, that $n$ and $k$ must have the same parity, see Section 2.) Define now the cohomology of $X$ graded cyclically as follows:

$$
\tilde{H}^{i}(X)=\bigoplus_{l \in \mathbb{Z}} H^{i+2 C_{X} l}(X), \quad \tilde{b}_{i}(X)=\operatorname{rank} \tilde{H}^{i}(X)
$$

Define $\tilde{H}^{i}(\Sigma)$ and $\tilde{b}_{i}(\Sigma)$ in a similar way (note that in the definition of $\tilde{H}^{i}(\Sigma)$ one has to replace also $C_{X}$ by $C_{\Sigma}$ ). Theorem A together with a simple application of the Lefschetz hyperplane section theorem give the following result: 
Corollary B. Let $X \subset \mathbb{C} P^{N}$ be an algebraic manifold with small dual and $b_{2}(X)=1$. Then $\tilde{b}_{j}(X)=\tilde{b}_{j+2}(X), \forall j \in \mathbb{Z}$. Moreover, if $\Sigma \subset X$ is a smooth hyperplane section then similarly to $X$, we have $\tilde{b}_{j}(\Sigma)=\tilde{b}_{j+2}(\Sigma), \forall j \in \mathbb{Z}$.

A similar result (for subcritical manifolds) has been previously obtained by He [29] using methods of contact homology.

If $\operatorname{dim}_{\mathbb{C}}(X)=n$ and $\operatorname{def}(X)=k$, Theorem B implies the following relations among the Betti numbers of $X$ :

$$
\begin{aligned}
b_{j}(X)+b_{j+n+k+2}(X) & =b_{j+2}(X)+b_{j+n+k+4}(X), \quad \forall 0 \leq j \leq n+k-1, \\
b_{n+k}(X) & =b_{n+k+2}(X)+1, \quad b_{n+k+1}(X)=b_{n+k+3}(X),
\end{aligned}
$$

and the following ones for those of $\Sigma$ :

$$
\begin{gathered}
b_{j}(\Sigma)+b_{j+n+k}(\Sigma)=b_{j+2}(\Sigma)+b_{j+n+k+2}(\Sigma), \quad \forall 0 \leq j \leq n+k-3, \\
b_{n+k-2}(\Sigma)=b_{n+k}(\Sigma)+1, \quad b_{n+k-1}(\Sigma)=b_{n+k+1}(\Sigma) .
\end{gathered}
$$

We will prove a slightly stronger result in Section 4, see Corollary 4.4 .

Example. Consider the complex Grassmannian $X=\mathrm{Gr}(5,2) \subset \mathbb{C} P^{9}$ of two-dimensional subspaces in $\mathbb{C}^{5}$ embedded in projective space by the Plücker embedding. It is known that $\operatorname{def}(X)=2$, see $[27,38,47]$. We have $\operatorname{dim}_{\mathbb{C}}(X)=6$ and $2 C_{X}=10$. The table of Betti numbers of $X$ is given as follows:

\begin{tabular}{|c|c|c|c|c|c|c|c|c|c|c|c|c|c|}
\hline $\mathrm{q}$ & 0 & 1 & 2 & 3 & 4 & 5 & 6 & 7 & 8 & 9 & 10 & 11 & 12 \\
\hline$b_{q}(X)$ & 1 & 0 & 1 & 0 & 2 & 0 & 2 & 0 & 2 & 0 & 1 & 0 & 1 \\
\hline
\end{tabular}

Further implications of Theorem A are obtained by studying the algebraic properties of the inverse $[\omega]^{-1}$. First note that due to degree reasons the inverse element should be of the form

$$
[\omega]^{-1}=\alpha_{n+k-2} \otimes q^{-1} \in \mathrm{OH}^{-2}(\Sigma ; \Lambda),
$$


where $\alpha_{n+k-2} \in H^{n+k-2}(\Sigma)$ is a nontrivial element. Moreover, this element needs to satisfy the following conditions:

$$
[\omega] \cup \alpha_{n+k-2}=0, \quad\left([\omega] * \alpha_{n+k-2}\right)_{1}=1,
$$

where $\left([\omega] * \alpha_{n+k-2}\right)_{1} \in H^{0}(\Sigma)$ is determined by the condition that

$$
\left\langle\left([\omega] * \alpha_{n+k-2}\right)_{1},-\right\rangle=\mathrm{GW}_{1}^{\Sigma}\left(\mathrm{PD}[\omega], \mathrm{PD}\left(\alpha_{n+k-2}\right),-\right) .
$$

Here PD stands for Poincaré duality, and for $a \in \mathrm{OH}^{l}(\Sigma ; \Lambda)$ and $i \in \mathbb{Z}$ we denote by $(a)_{i} \in$ $H^{l-2 i C_{\Sigma}}(\Sigma)$ the coefficient of $q^{i}$ in $a$. The notation $\mathrm{GW}_{j}^{\Sigma}(A, B, C)$ stands for the GromovWitten invariant counting the number of rational curves $u: \mathbb{C} P^{1} \rightarrow \Sigma$ passing through three cycles representing the homology classes $A, B$, and $C$ with $C_{1}\left(u_{*}\left[\mathbb{C} P^{1}\right]\right)=j C_{\Sigma}$.

So in our case, the fact that $\left([\omega] * \alpha_{n+k-2}\right)_{1} \neq 0$ implies that $\Sigma$ is uniruled. The uniruldness of $\Sigma$ (as well as that of $X$ ) was previously known and the variety of rational curves on it was studied by Ein [21]. Finally, note that the uniruldness of $X$ follows also from the results of $\mathrm{He}$ [29] in combination with Theorem 6.1.

The method of proof of Theorem A is an application of the theory of Hamiltonian fibrations and, in particular, their Seidel elements, see [44]. In [44], Seidel constructed a representation of $\pi_{1}(\operatorname{Ham}(\Sigma, \omega))$ on $\mathrm{OH}(\Sigma ; \Lambda)$ given by a group homomorphism

$$
S: \pi_{1}(\operatorname{Ham}(\Sigma, \omega)) \longrightarrow \mathrm{OH}(\Sigma ; \Lambda)^{\times}
$$

where $\operatorname{OH}(\Sigma ; \Lambda)^{\times}$is the group of invertible elements of the quantum cohomology algebra.

Theorem A follows from:

Theorem C. Let $X \subset \mathbb{C} P^{N}$ be an algebraic manifold with small dual and $b_{2}(X)=1$. Let $\Sigma \subset X$ be a smooth hyperplane section of $X$ and denote by $\omega$ the symplectic structure induced on $\Sigma$ from $\mathbb{C} P^{N}$. There exists a nontrivial element $1 \neq \lambda \in \pi_{1}(\operatorname{Ham}(\Sigma, \omega))$ whose Seidel element is given by

$$
S(\lambda)=[\omega] \in \mathrm{QH}^{2}(\Sigma ; \Lambda)
$$

See Theorems 4.2 and 9.1 for more general statements.

Before we turn to examples, let us mention that by results of [4], based on Mori theory, the classification of manifolds with small dual is reduced to the case $b_{2}(X)=1$. 
Here is a list of examples of manifolds with small dual and $b_{2}(X)=1$ (see [47] for more details):

\section{Examples.}

(1) $X=\mathbb{C} P^{n} \subset \mathbb{C} P^{n+1}$ has $\operatorname{def}(X)=n$.

(2) $X=\operatorname{Gr}(2 l+1,2)$ embedded via the Plücker embedding has $\operatorname{def}(X)=2$. $($ See $[27,38,47]$.

(3) $X=\mathbb{S}_{5} \subset \mathbb{C} P^{15}$ the 10-dimensional spinor variety has $\operatorname{def}(X)=4$. (See [34, 47].)

(4) In any of the examples (1)-(3), one can take iterated hyperplane sections and still obtain manifolds with def $>0$ and $b_{2}=1$, provided that the number of iterations does not exceed the defect-1. (See Section 2.)

The manifolds in (1)-(3) together with the corresponding hyperplane sections (4) are the only known examples of projective manifolds with small dual and $b_{2}(X)=1$, see $[5,45]$. On the basis of these examples, it is conjectured in [5] that all nonlinear algebraic manifolds with $b_{2}(X)=1$ have $\operatorname{def}(X) \leq 4$.

Organization of the paper. The rest of the paper is organized as follows: In Section 2, we recall basic facts on projective manifolds with small dual. In Section 3, we review relevant results from the theory of Hamiltonian fibrations and the Seidel representation. In Section 4, we explain the relation between manifolds with small dual and Hamiltonian fibrations. In Section 5, we prove Theorems A and C. In Section 6, we discuss the relation between manifolds with small dual and subcritical Stein manifolds and derive some topological consequences from that. Corollary B is proved in Section 7. In Section 8, we present more applications of our methods to questions on the symplectic topology and algebraic geometry of manifolds with small dual. We also outline an alternative proof of Corollary B based on Lagrangian Floer theory. In Section 9, we explain how to generalize Theorem A to the case $b_{2}(X)>1$ (or more generally to nonmonotone manifolds). In the same section, we also work out explicitly such an example. Finally, in Section 10, we discuss some open questions and further possible directions of study.

\section{Basic Results on Projective Manifolds with Small Dual}

Let $X \subset \mathbb{C} P^{N}$ be an algebraic manifold of $\operatorname{dim}_{\mathbb{C}} X=n$. Denote by $\left(\mathbb{C} P^{N}\right)^{*}$ the dual projective space parametrizing hyperplanes $H \subset \mathbb{C} P^{N}$. To $X$ one associates the dual 
variety $X^{*} \subset\left(\mathbb{C} P^{N}\right)^{*}$, which (in the case $X$ is smooth) is defined as

$$
X^{*}=\{H \mid H \text { is somewhere tangent to } X\} \text {. }
$$

We refer the reader to [47] for a detailed account on the subject of projective duality. In this section, we will review basic properties of projective manifolds with positive defect. Define the defect of $X$ to be

$$
\operatorname{def}(X)=\operatorname{codim}_{\mathbb{C}} X^{*}-1
$$

Note that when $X^{*}$ is a hypersurface the defect of $X$ is zero. An important feature of the defect is the following: if $\operatorname{def}(X)=k$ then for a smooth point of the dual variety, $H \in X_{\mathrm{sm}}^{*}$, the singular part $\operatorname{sing}(X \cap H)$ of $X \cap H$ is a projective space of dimension $k$ linearly embedded in $\mathbb{C} P^{N}$. Thus, $X$ is covered by projective spaces of dimension $k$, and, in particular, there is a projective line through every point of $X$ (see [30]).

Next, the defect of $X$ and that of a hyperplane section $\Sigma \subset X$ of $X$ are related as follows (see [20]):

$$
\operatorname{def}(\Sigma)=\max \{\operatorname{def}(X)-1,0\}
$$

A well-known (unpublished) result of Landman states that for manifolds $X$ with small dual we have the following congruence $\operatorname{dim}_{\mathbb{C}}(X) \equiv \operatorname{def}(X)(\bmod 2)$ (see $[21,47]$ for a proof of this).

Later, Ein proved in [21] the following. Let $X \subset \mathbb{C} P^{N}$ be an algebraic manifold with $\operatorname{dim}_{\mathbb{C}}(X)=n$ and $\operatorname{def}(X)=k>0$. Denote by $c_{1}^{X}$ the first Chern class of $X$. Then through every point in $X$ there exists a projective line $S$ with

$$
c_{1}^{X}(S)=\frac{n+k}{2}+1
$$

Of special importance is the case $b_{2}(X)=1$, which was extensively studied by Ein in [21]. In this case, we have:

$$
c_{1}^{X}=\left(\frac{n+k}{2}+1\right) \cdot h
$$

where $h \in H^{2}(X) \cong \mathbb{Z}$ is the positive generator, which is also the class of the restriction (to $X$ ) of the Kähler form of $\mathbb{C} P^{N}$. In particular, in this case both $X$ and $\Sigma$ are Fano manifolds. 


\section{Hamiltonian Fibrations}

In what follows, we will use the theory of symplectic and Hamiltonian fibrations and their invariants. We refer the reader to [28, 36, 37] for the foundations.

Let $\pi: \tilde{X} \rightarrow B$ be a smooth locally trivial fibration with fiber $\Sigma$ and base $B$ which are both closed manifolds. We will assume in addition that $B$ is a simply connected manifold. Further, let $\tilde{\Omega}$ be a closed 2 -form on $\tilde{X}$ such that the restriction $\Omega_{b}=\left.\tilde{\Omega}\right|_{\Sigma_{b}}$ to each fiber $\Sigma_{b}=\pi^{-1}(b), b \in B$, is a symplectic form. Fix $b_{0} \in B$, and let $\omega_{\Sigma}$ be a symplectic form on $\Sigma$ such that $\left(\Sigma, \omega_{\Sigma}\right)$ is symplectomorphic to $\left(\Sigma_{b_{0}}, \Omega_{b_{0}}\right)$. This structure is a special case of a so-called Hamiltonian fibration. It is well known that under these assumptions all fibers $\left(\Sigma_{b}, \Omega_{b}\right)$ are symplectomorphic and in fact the structure group of $\pi$ can be reduced to $\operatorname{Ham}\left(\Sigma, \omega_{\Sigma}\right)$.

We will assume from now on that $B=S^{2}$. We identify $S^{2} \cong \mathbb{C} P^{1}$ in a standard way and view $S^{2}$ as a Riemann surface whose complex structure is denoted by $j$.

\subsection{Holomorphic curves in Hamiltonian fibrations}

Let $\pi:(\tilde{X}, \tilde{\Omega}) \rightarrow S^{2}$ be a Hamiltonian fibration as above. Denote by $T^{v} \tilde{X}=\operatorname{ker}(D \pi)$ the vertical part of the tangent bundle of $\tilde{X}$. We now introduce almost complex structures compatible with the fibration. These are by definition almost complex structures $\tilde{J}$ on $\tilde{X}$ with the following properties:

(1) The projection $\pi$ is $(\tilde{J}, j)$-holomorphic.

(2) For every $z \in S^{2}$ the restriction $J_{z}$ of $\tilde{J}$ to $\Sigma_{z}$ is compatible with the symplectic form $\Omega_{z}$, that is, $\Omega_{z}\left(J_{z} \xi, J_{z} \eta\right)=\Omega_{z}(\xi, \eta)$ for every $\xi, \eta \in T_{z}^{v} \tilde{X}$, and $\Omega_{z}\left(\xi, J_{z} \xi\right)>0$ for every $0 \neq \xi \in T_{z}^{v} \tilde{X}$.

We denote the space of such almost complex structures by $\tilde{\mathcal{J}}(\pi, \tilde{\Omega})$.

Denote by $H_{2}^{\pi} \subset H_{2}(\tilde{X} ; \mathbb{Z})$ the set of classes $\tilde{A}$ such that $\pi_{*}(\tilde{A})=\left[S^{2}\right]$. Given $\tilde{A}$ and $\tilde{J} \in \tilde{\mathcal{J}}(\pi, \tilde{\Omega})$ denote by $\mathcal{M}^{\mathfrak{s}}(\tilde{A}, \tilde{J})$ the space of $\tilde{J}$-holomorphic sections in the class $\tilde{A}$, that is, the space of maps $\tilde{u}: S^{2} \longrightarrow \tilde{X}$ with the following properties:

(1) $\tilde{u}$ is $(j, \tilde{J})$-holomorphic.

(2) $\tilde{u}$ is a section, that is, $\pi \circ \tilde{u}=\mathrm{id}$.

(3) $\tilde{u}_{*}\left[S^{2}\right]=\tilde{A}$.

Fix $z_{0} \in S^{2}$ and fix an identification $\left(\Sigma, \omega_{\Sigma}\right) \approx\left(\Sigma_{z_{0}}, \Omega_{z_{0}}\right)$. The space of sections comes with an evaluation map:

$$
e v_{\tilde{J}, z_{0}}: \mathcal{M}^{\mathfrak{s}}(\tilde{A}, \tilde{J}) \longrightarrow \Sigma, \quad e v_{\tilde{J}, z_{0}}(\tilde{u})=\tilde{u}\left(z_{0}\right)
$$




\subsubsection{Transversality}

In order to obtain regularity and transversality properties for the moduli spaces of holomorphic sections and their evaluation maps, we will need to work with so-called regular almost complex structures. Moreover, since the moduli spaces of holomorphic sections are usually not compact they do not carry fundamental classes and so the evaluation maps do not induce in a straightforward way homology classes in their target ( $\Sigma$ in this case). The reason for noncompactness of these moduli spaces is that a sequence of holomorphic sections might develop bubbles in one of the fibers (see, e.g., [37]). The simplest way to overcome this difficulty is to make some positivity assumptions on the fiber $\Sigma$ (called monotonicity). Under such conditions, the moduli spaces of holomorphic sections admits a nice compactification which makes it possible to define homology classes induced by the evaluation maps. Here is the relevant definition.

Definition 3.1. Let $\left(\Sigma, \omega_{\Sigma}\right)$ be a symplectic manifold. Denote by $H_{2}^{S}(\Sigma) \subset H_{2}(\Sigma$; $\mathbb{Z})$ the image of the Hurewicz homomorphism $\pi_{2}(\Sigma) \longrightarrow H_{2}(\Sigma ; \mathbb{Z})$. Denote by $c_{1}^{\Sigma} \in H^{2}(\Sigma ; \mathbb{Z})$ the first Chern class of the tangent bundle $\left(T \Sigma, J_{\Sigma}\right)$, where $J_{\Sigma}$ is any almost complex structure compatible with $\omega_{\Sigma}$. The symplectic manifold $\left(\Sigma, \omega_{\Sigma}\right)$ is called spherically monotone if there exists a constant $\lambda>0$ such that for every $A \in H_{2}^{S}(\Sigma)$ we have $\omega_{\Sigma}(A)=\lambda c_{1}^{\Sigma}(A)$. For example, if $\Sigma$ is a Fano manifold and $\omega_{\Sigma}$ is a symplectic form with $\left[\omega_{\Sigma}\right]=c_{1}^{\Sigma}$ then obviously $\left(\Sigma, \omega_{\Sigma}\right)$ is spherically monotone.

From now on, we assume that the fiber $\left(\Sigma, \omega_{\Sigma}\right)$ of $\pi:(\tilde{X}, \tilde{\Omega}) \longrightarrow S^{2}$ is spherically monotone. Denote by $c_{1}^{v}=c_{1}\left(T^{v} \tilde{X}\right) \in H^{2}(\tilde{X})$ the vertical Chern class, that is, the first Chern class of the vertical tangent bundle of $\tilde{X}$. The following is proved in [37,44]. There exists a dense subset $\tilde{\mathcal{J}}_{\text {reg }}(\pi, \tilde{\Omega}) \subset \tilde{\mathcal{J}}(\pi, \tilde{\Omega})$ such that for every $\tilde{J} \in \tilde{\mathcal{J}}_{\text {reg }}(\pi, \tilde{\Omega})$ and every $\tilde{A} \in H_{2}^{\pi}$ the following holds:

(1) For every $\tilde{A} \in H_{2}^{\pi}$, the moduli space $\mathcal{M}^{\mathfrak{s}}(\tilde{A}, \tilde{J})$ of $\tilde{J}$-holomorphic sections in the class $\tilde{A}$ is either empty or a smooth manifold of dimension

$$
\operatorname{dim}_{\mathbb{R}} \mathcal{M}^{\mathfrak{s}}(\tilde{A}, \tilde{J})=\operatorname{dim}_{\mathbb{R}} \Sigma+2 c_{1}^{v}(\tilde{A})
$$

Moreover, $\mathcal{M}^{\mathfrak{s}}(\tilde{A}, \tilde{J})$ has a canonical orientation.

(2) The evaluation map $e v_{\tilde{J}, Z_{0}}: \mathcal{M}^{\mathfrak{s}}(\tilde{A}, \tilde{J}) \longrightarrow \Sigma$ is a pseudo-cycle (see [37] for the definition). In particular, its Poincaré dual gives a cohomology class $\mathcal{S}(\tilde{A} ; \tilde{J}) \in H^{d}(\Sigma ; \mathbb{Z})_{\text {free }}=H^{d}(\Sigma ; \mathbb{Z}) /$ torsion, where $d=-2 c_{1}^{v}(\tilde{A})$. Moreover, the 
class $\mathcal{S}(\tilde{A} ; \tilde{J})$ is independent of the regular $\tilde{J}$ used to define it. Therefore, we will denote it from now on by $\mathcal{S}(\tilde{A})$.

We refer the reader to $[37,44]$ for more general results on transversality.

The definition of regularity for $\tilde{J} \in \tilde{\mathcal{J}}(\pi, \tilde{\Omega})$ involves three ingredients. The first is that the restriction $J_{z_{0}}$ of $\tilde{J}$ to $\Sigma=\Sigma_{z_{0}}$ is regular in the sense of [37, Chapter 3], namely that the linearization of the $\bar{\partial}_{J_{z_{0}}}$-operator at every $J_{z_{0}}$-holomorphic curve in $\Sigma$ is surjective. (In addition one has to require that certain evaluation maps for tuples of such curves are mutually transverse.) The second ingredient is that (the vertical part of) the $\bar{\partial}_{\tilde{J}}$-operator at every $\tilde{J}$-holomorphic section is surjective. The third one is that $e v_{\tilde{J}, z_{0}}$ is transverse to all $J_{z_{0}}$-holomorphic bubble trees in $\Sigma$.

In practice, we will have to compute cohomology classes of the type $\mathcal{S}(\tilde{A})=$ $\mathcal{S}(\tilde{A} ; \tilde{J})$ using a specific choice of $\tilde{J}$ that naturally appears in our context. It is not an easy task to decide whether a given almost complex structure $\tilde{J}$ is regular or not. However, in some situations, it is possible to compute some of the classes $\mathcal{S}(\tilde{A})$ by using almost complex structures $\tilde{J}$ that satisfy weaker conditions than regularity. Criteria for verification of these conditions have been developed in [44] (see Proposition 7.11 there) and in [37] (see Sections 3.3 and 3.4). Below, we will actually not appeal to such criteria and use simpler arguments.

\subsection{The Seidel representation}

Let $\left(\Sigma, \omega_{\Sigma}\right)$ be a closed monotone symplectic manifold (see Definition 3.1 is Section 3.1.1). Denote by $C_{\Sigma} \in \mathbb{N}$ the minimal Chern number, that is,

$$
C_{\Sigma}=\min \left\{C_{1}^{\Sigma}(A) \mid A \in H_{2}^{S}, C_{1}^{\Sigma}(A)>0\right\}
$$

Denote by $\Lambda=\mathbb{Z}\left[q^{-1}, q\right]$ the ring of Laurent polynomials. We endow $\Lambda$ with a grading by setting $\operatorname{deg}(q)=2 C_{\Sigma}$. Let $\mathrm{OH}^{*}(\Sigma ; \Lambda)=\left(H^{\bullet}(\Sigma) \otimes \Lambda\right)^{*}$ be the quantum cohomology of $\Sigma$, where the grading is induced from both factors $H^{\bullet}(\Sigma)$ and $\Lambda$. We endow $\mathrm{OH}(\Sigma ; \Lambda)$ with the quantum product $*$. The unity will be denoted as usual by $1 \in \mathrm{OH}^{0}(\Sigma ; \Lambda)$. We refer the reader to [37, Chapter 11] for the definitions and foundations of quantum cohomology. (Note, however, that our grading conventions are slightly different than the ones in [37].) With our grading conventions, we have:

$$
\mathrm{OH}^{j}(\Sigma ; \Lambda)=\bigoplus_{l \in \mathbb{Z}} H^{j-2 l C_{\Sigma}}(\Sigma) q^{l} .
$$


We will also need a coefficient extension of $\mathrm{OH}(\Sigma ; \Lambda)$. Denote by $\bar{\Lambda}=\mathbb{Z}\left[t^{-1}, t\right]$ the ring of Laurent polynomials in the variable $t$, graded so that $\operatorname{deg}(t)=2$. Consider now $\mathrm{OH}^{*}(\Sigma ; \bar{\Lambda})=\left(H^{\bullet}(\Sigma) \otimes \bar{\Lambda}\right)^{*}$, endowed with the quantum product $*$. We can regard $\bar{\Lambda}$ as an algebra over $\Lambda$ using the embedding of rings induced by $q \longmapsto t^{C_{\Sigma}}$. This also induces an embedding of rings

$$
\mathrm{OH}^{*}(\Sigma ; \Lambda) \longleftrightarrow \mathrm{OH}^{*}(\Sigma ; \bar{\Lambda})
$$

We will, therefore, view from now on $\mathrm{OH}(\Sigma ; \Lambda)$ as a subring of $\mathrm{OH}(\Sigma ; \bar{\Lambda})$ by setting $q=t^{C_{\Sigma}}$.

In [44], Seidel associated to a Hamiltonian fibration $\pi: \tilde{X} \longrightarrow S^{2}$ with fiber $\Sigma$ an invertible element $\tilde{S}(\pi) \in \mathrm{OH}^{0}(\Sigma ; \bar{\Lambda})$. We refer the reader to [37,44] for a detailed account of this theory. Here is a brief review of the main construction.

Pick a regular almost complex structure $\tilde{J} \in \tilde{\mathcal{J}}_{\text {reg }}(\pi, \tilde{\Omega})$. Define a class:

$$
\tilde{S}(\pi):=\sum_{\tilde{A} \in H_{2}^{\pi}} \mathcal{S}(\tilde{A} ; \tilde{J}) \otimes t^{C_{1}^{v}(\tilde{A})} \in \mathrm{OH}^{0}(\Sigma ; \bar{\Lambda}) .
$$

Note that since the degree of $\mathcal{S}(\tilde{A}, \tilde{J})$ is $-2 c_{1}^{v}(\tilde{A})$, a class $\tilde{A} \in H_{2}^{\pi}$ contributes to the sum in (4) only if

$$
2-2 n \leq 2 c_{1}^{v}(\tilde{A}) \leq 0
$$

The class $\tilde{S}(\pi)$ is called the Seidel element of the fibration $\pi:(\tilde{X}, \tilde{\Omega}) \longrightarrow S^{2}$.

In what follows, it will be more convenient to work with the more "economical" ring $\Lambda$ rather than $\bar{\Lambda}$. We will now define a normalized version of the Seidel element, denoted $S(\pi)$, which lives in $\operatorname{QH}(\Sigma ; \Lambda)$. Fix a reference class $\tilde{A}_{0} \in H_{2}^{\pi}$ and set $c_{0}(\pi)=$ $c_{1}^{v}\left(\tilde{A}_{0}\right)$. Define now

$$
S(\pi)=t^{-C_{0}(\pi)} \tilde{S}(\pi)
$$

Since any two classes in $H_{2}^{\pi}$ differ by a class in $H_{2}^{S}(\Sigma)$, there exists a uniquely defined function $v: H_{2}^{\pi} \rightarrow \mathbb{Z}$ such that

$$
C_{1}^{v}(\tilde{A})=c_{0}(\pi)+v(\tilde{A}) C_{\Sigma}, \quad \forall \tilde{A} \in H_{2}^{\pi}
$$

As $q=t^{C_{\Sigma}}$ we have

$$
S(\pi):=\sum_{\tilde{A} \in H_{2}^{\pi}} \mathcal{S}(\tilde{A} ; \tilde{J}) \otimes q^{\nu(\tilde{A})} \in \mathrm{OH}^{-2 c_{0}(\pi)}(\Sigma ; \Lambda)
$$


By abuse of terminology, we will call $S(\pi)$ also the Seidel element of the fibration $\pi$. Of course the element $S(\pi)$ (as well as its degree) depends on the choice of the reference section $\tilde{A}_{0}$, however, different reference sections $\tilde{A}_{0}$ will result in elements that differ by a factor of the type $q^{r}$ for some $r \in \mathbb{Z}$. In particular, many algebraic properties of $S(\pi)$ (such as invertibility) do not depend on this choice. We will therefore ignore this ambiguity from now on.

\subsubsection{Relations to Hamiltonian loops}

An important feature of the theory is the connection between Hamiltonian fibrations over $S^{2}$ with fiber $\left(\Sigma, \omega_{\Sigma}\right)$ and $\pi_{1}\left(\operatorname{Ham}\left(\Sigma, \omega_{\Sigma}\right)\right)$. To a loop based at the identity $\lambda=\left\{\varphi_{t}\right\}_{t \in S^{1}}$ in $\operatorname{Ham}\left(\Sigma, \omega_{\Sigma}\right)$ one can associate a Hamiltonian fibration $\pi_{\lambda}: \tilde{M}_{\lambda} \rightarrow S^{2}$ as follows. Let $D_{+}$ and $D_{-}$be two copies of the unit disk in $\mathbb{C}$, where the orientation on $D_{-}$is reversed. Define:

$$
\tilde{M}_{\lambda}=\left(\left(\Sigma \times D_{+}\right) \coprod\left(\Sigma \times D_{-}\right)\right) / \sim \quad \text { where }\left(x, e_{+}^{2 \pi i t}\right) \sim\left(\varphi_{t}(x), e_{-}^{2 \pi i t}\right) .
$$

Identifying $S^{2} \approx D_{+} \cup_{\partial} D_{-}$we obtain a fibration $\pi: \tilde{M}_{\lambda} \longrightarrow S^{2}$. As the elements of $\lambda$ are symplectic diffeomorphisms, the form $\omega_{\Sigma}$ gives rise to a family of symplectic forms $\left\{\Omega_{z}\right\}_{z \in S^{2}}$ on the fibers $\Sigma_{z}=\pi^{-1}(z)$ of $\pi$. Moreover, $\pi:\left(\tilde{M}_{\lambda},\left\{\Omega_{z}\right\}_{z \in S^{2}}\right) \longrightarrow S^{2}$ is locally trivial. Since the elements of $\lambda$ are in fact Hamiltonian diffeomorphisms it follows that the family of fiberwise forms $\left\{\omega_{z}\right\}_{z \in S^{2}}$ can be extended to a closed 2-form $\tilde{\Omega}$ on $\tilde{M}_{\lambda}$, that is, $\left.\tilde{\Omega}\right|_{\Sigma_{z}}=\Omega_{z}$ for every $z$. See $[37,44]$ for the proofs. We therefore obtain from this construction a Hamiltonian fibration $\pi:\left(\tilde{M}_{\lambda}, \tilde{\Omega}\right) \longrightarrow S^{2}$.

From the construction, one can see that homotopic loops in $\operatorname{Ham}\left(\Sigma, \omega_{\Sigma}\right)$, give rise to isomorphic fibrations. We denote the isomorphism class of fibrations corresponding to an element $\gamma \in \pi_{1}\left(\operatorname{Ham}\left(\Sigma, \omega_{\Sigma}\right)\right)$ by $\pi_{\gamma}$.

Conversely, if $\pi:(\tilde{M}, \tilde{\Omega}) \longrightarrow S^{2}$ is a Hamiltonian fibration with fiber $\left(\Sigma, \omega_{\Sigma}\right)$ one can express $\tilde{M}$ as a gluing of two trivial bundles over the two hemispheres in $S^{2}$. The gluing map would be a loop of Hamiltonian diffeomorphisms of $\left(\Sigma, \omega_{\Sigma}\right)$. Different trivializations lead to homotopic loops. Thus, the fibration $\pi$ determines a class $\gamma(\pi) \in \pi_{1}\left(\operatorname{Ham}\left(\Sigma, \omega_{\Sigma}\right)\right)$.

This correspondence has the following properties in relation to the Seidel elements (see [44] for the proofs):

$$
\bar{S}\left(\pi_{\gamma_{1} \cdot \gamma_{2}}\right)=\bar{S}\left(\pi_{\gamma_{1}}\right) * \bar{S}\left(\pi_{\gamma_{2}}\right) \quad \forall \gamma_{1}, \gamma_{2} \in \pi_{1}\left(\operatorname{Ham}\left(\Sigma, \omega_{\Sigma}\right)\right) .
$$


Here $*$ stands for the quantum product. The unit element $e \in \pi_{1}\left(\operatorname{Ham}\left(\Sigma, \omega_{\Sigma}\right)\right)$ corresponds to the trivial fibration $\pi_{e}: \Sigma \times S^{2} \longrightarrow S^{2}$ and we have $\bar{S}\left(\pi_{e}\right)=1 \in \mathrm{QH}(\Sigma ; \Lambda)$. It follows that $\bar{S}(\pi)$ is an invertible element in $\mathrm{QH}(\Sigma ; \bar{\Lambda})$ for every $\pi$. The corresponding homomorphism

$$
\bar{S}: \pi_{1}\left(\operatorname{Ham}\left(\Sigma, \omega_{\Sigma}\right)\right) \longrightarrow \mathrm{OH}(\Sigma, \bar{\Lambda})^{\times}, \quad \gamma \longmapsto \bar{S}\left(\pi_{\gamma}\right)
$$

(which by abuse of notation we also denote by $\bar{S}$ ), where $\mathrm{OH}(\Sigma, \bar{\Lambda})^{\times}$is the group of invertible elements in $\mathrm{OH}(\Sigma, \bar{\Lambda})$, is called the Seidel representation.

As mentioned before, for our purposes it would be more convenient to work with the normalized version $S(\pi)$ of the Seidel element rather than with $\bar{S}(\pi)$. We claim that any normalized Seidel element $S(\pi)$ is invertible in $\mathrm{OH}(\Sigma ; \Lambda)$ (not just in $\mathrm{OH}(\Sigma ; \bar{\Lambda})$ ). To see this, denote by $\gamma \in \pi_{1}(\operatorname{Ham}(\Sigma))$ the homotopy class of loops corresponding to the fibration $\pi$ (so that $\pi=\pi_{\gamma}$ ). Denote by $\pi^{\prime}=\pi_{\gamma^{-1}}$ the fibration corresponding to the inverse of $\gamma$. Choose two reference sections $\tilde{A}_{0}$ and $\widetilde{A}_{0}^{\prime}$ for $\pi$ and $\pi^{\prime}$, respectively. The corresponding normalized Seidel elements are $S(\pi)=t^{-C_{0}(\pi)} \tilde{S}(\pi), S\left(\pi^{\prime}\right)=t^{-C_{0}\left(\pi^{\prime}\right)} \tilde{S}\left(\pi^{\prime}\right)$. Since $\tilde{S}_{\pi} * \tilde{S}_{\pi^{\prime}}=1$, we have

$$
S(\pi) * S\left(\pi^{\prime}\right)=t^{-c_{0}(\pi)-c_{0}\left(\pi^{\prime}\right)}
$$

But $S(\pi)$ and $S\left(\pi^{\prime}\right)$ both belong to the subring $\mathrm{OH}(\Sigma ; \Lambda)$ of $\mathrm{OH}(\Sigma ; \bar{\Lambda})$, hence their product $S(\pi) * S\left(\pi^{\prime}\right) \in \mathrm{OH}(\Sigma ; \Lambda)$ too. Thus, $t^{-c_{0}(\pi)-c_{0}\left(\pi^{\prime}\right)}=q^{r}$ for some $r \in \mathbb{Z}$. It follows that $S(\pi)$ is invertible in $\mathrm{QH}(\Sigma ; \Lambda)$.

\section{From Manifolds with Small Dual to Hamiltonian Fibrations}

Let $X \subset \mathbb{C} P^{N}$ be a projective manifold with small dual. Put $n=\operatorname{dim}_{\mathbb{C}} X$ and $k=\operatorname{def}(X)>$ 0 . Since $X^{*} \subset\left(\mathbb{C} P^{N}\right)^{*}$ has codimension $k+1 \geq 2$, we can find a pencil of hyperplanes $\ell \subset\left(\mathbb{C} P^{N}\right)^{*}$ such that $\ell$ does not intersect $X^{*}$. Consider the manifold

$$
\tilde{X}=\{(x, H) \mid H \in \ell, x \in H\} \subset X \times \ell .
$$

Identify $\ell \cong \mathbb{C} P^{1} \cong S^{2}$ in an obvious way. Denote by

$$
p: \tilde{X} \longrightarrow X, \quad \pi_{\ell}: \tilde{X} \longrightarrow \ell \cong S^{2}
$$


the obvious projections. The map $p$ can be considered as the blowup of $X$ along the base locus of the pencil $\ell$. The map $\pi_{\ell}$ is a honest holomorphic fibration (without singularities) over $\ell \cong \mathbb{C} P^{1}$ with fibers $\pi_{\ell}^{-1}(H)=X \cap H$.

Denote by $\omega_{X}$ the symplectic form on $X$ induced from the Fubini-Study Kähler form of $\mathbb{C} P^{N}$. Let $\omega_{S^{2}}$ be an area form on $S^{2}$ with $\int_{S^{2}} \omega_{S^{2}}=1$. Endow $X \times S^{2}$ with $\omega_{X} \oplus \omega_{S^{2}}$ and denote by $\tilde{\Omega}$ the restriction of $\omega_{X} \oplus \omega_{S^{2}}$ to $\tilde{X} \subset X \times S^{2}$. The restriction of $\tilde{\Omega}$ to the fibers $\left.\tilde{\Omega}\right|_{\pi_{\ell}^{-1}(H)}, H \in \ell$, coincides with the symplectic forms $\left.\omega_{X}\right|_{X \cap H}$. Thus, $\pi_{\ell}: \tilde{X} \longrightarrow S^{2}$ is a Hamiltonian fibration. Fix a point $H_{0} \in \ell$, and set $\left(\Sigma, \omega_{\Sigma}\right)=\left(\pi_{\ell}^{-1}\left(H_{0}\right),\left.\omega_{X}\right|_{X \cap H_{0}}\right)$.

Remark 4.1. Different pencils $\ell \subset\left(\mathbb{C} P^{N}\right)^{*}$ with $\ell \cap X^{*}=\emptyset$ give rise to isomorphic Hamiltonian fibrations. This is so because the real codimension of $X^{*}$ is at least 4 hence any two pencils $\ell$ and $\ell^{\prime}$ which do not intersect $X^{*}$ can be connected by a real path of pencils in the complement of $X^{*}$. Thus, the isomorphism class of the Hamiltonian fibration $\pi_{\ell}$, the element $\gamma\left(\pi_{\ell}\right) \in \pi_{1}\left(\operatorname{Ham}\left(\Sigma, \omega_{\Sigma}\right)\right)$, as well as the corresponding Seidel element $S\left(\pi_{\ell}\right)$ can all be viewed as invariants of the projective embedding $X \subset \mathbb{C} P^{N}$.

Theorem 4.2. Let $X \subset \mathbb{C} P^{N}$ be an algebraic manifold with $\operatorname{dim}_{\mathbb{C}}(X)=n \geq 2$ and $\operatorname{def}(X)=k>0$. Denote by $H_{2}^{S}(X)=\operatorname{image}\left(\pi_{2}(X) \longrightarrow H_{2}(X ; \mathbb{Z})\right) \subset H_{2}(X ; \mathbb{Z})$ the image of the Hurewicz homomorphism. Denote by $h \in H^{2}(X)$ the class dual to the hyperplane section. Assume that there exists $0<\lambda \in \mathbb{Q}$ such that $c_{1}^{X}(A)=\lambda h(A)$ for every $A \in H_{2}^{S}(X)$. Then the Seidel element of the fibration $\pi_{\ell}: \tilde{X} \longrightarrow \ell$ is

$$
S\left(\pi_{\ell}\right)=\left[\omega_{\Sigma}\right] \in \mathrm{OH}^{2}(\Sigma ; \Lambda) .
$$

The degree of the variable $q \in \Lambda$ is $\operatorname{deg}(q)=\frac{n+k}{2}$.

The proof of this Theorem is given in Section 5.

Remark 4.3. The condition $c_{1}^{X}(A)=\lambda h(A), \forall A \in H_{2}^{S}$, implies that $\lambda=\frac{n+k+2}{2}$. Indeed, as explained in Section 2, manifolds $X$ with small dual contain projective lines $S \subset X$ (embedded linearly in $\mathbb{C} P^{N}$ ) with $c_{1}^{X}(S)=\frac{n+k+2}{2}$. As $h(S)=1$ it follows that $\lambda=\frac{n+k+2}{2}$.

Examples. Theorem 4.2 applies for example to algebraic manifolds $X \subset \mathbb{C} P^{N}$ with small dual that satisfy one of the following conditions:

(1) $b_{2}(X)=1$

(2) More generally, the free part of $H_{2}^{S}(X)$ has rank 1 . 
This is so because in both of these cases we must have $h=\lambda c_{1}^{X}$ for some $\lambda \in \mathbb{Q}$. The fact that $\lambda>0$ follows from the existence of rational curves $S \subset X$ with $C_{1}^{X}(S)=\frac{n+k+2}{2}$ as explained in Section 2.

Here is a concrete class of examples with $b_{2}(X)>1$ (hence different than those in Section 1) to which Theorem 4.2 applies. Let $Y \subset \mathbb{C} P^{m}$ be any algebraic manifold with $\pi_{2}(Y)=0$ (or more generally with $h_{Y}(A)=0$ for every $A \in H_{2}^{S}(Y)$, where $h_{Y}$ is the Poincaré dual of the hyperplane class on $Y)$. Let $i: \mathbb{C} P^{n} \times \mathbb{C} P^{m} \longrightarrow \mathbb{C} P^{(n+1)(m+1)-1}$ be the Segre embedding and put $X=i\left(\mathbb{C} P^{n} \times Y\right)$. It is well known (see [46, Theorem 6.5]) that

$$
\operatorname{def}(X) \geq n-\operatorname{dim}_{\mathbb{C}}(Y)
$$

hence if $n>\operatorname{dim}_{\mathbb{C}}(Y), X$ will have a small dual. Note that the conditions of Theorem 4.2 are obviously satisfied.

One could generalize this example further by replacing $\mathbb{C} P^{n}$ with any manifold $Z$ satisfying $C_{1}^{Z}(A)=\lambda h_{Z}(A)$ for every $A \in H_{2}^{S}(Z)$ for some $\lambda>0$ and such that $\operatorname{def}(Z)>$ $\operatorname{dim}_{\mathbb{C}}(Y)$. (See [46] for more on such examples.)

Corollary 4.4. Under the assumptions of Theorem 4.2, we have

$$
\tilde{b}_{j}(X)=\tilde{b}_{j+2}(X), \quad \tilde{b}_{j}(\Sigma)=\tilde{b}_{j+2}(\Sigma) \quad \forall j \in \mathbb{Z},
$$

where the definition of $\tilde{b}_{j}$ is given in (1) in Section 1. Or, put in an unwrapped way, we have the following identities for $X$ :

$$
\begin{aligned}
b_{j}(X)+b_{j+n+k+2}(X) & =b_{j+2}(X)+b_{j+n+k+4}(X), \quad \forall 0 \leq j \leq n+k-1, \\
b_{n+k}(X) & =b_{n+k+2}(X)+1, \quad b_{n+k+1}(X)=b_{n+k+3}(X)+b_{1}(X),
\end{aligned}
$$

and the following ones for $\Sigma$ :

$$
\begin{aligned}
b_{j}(\Sigma)+b_{j+n+k}(\Sigma) & =b_{j+2}(\Sigma)+b_{j+n+k+2}(\Sigma), \quad \forall 0 \leq j \leq n+k-3, \\
b_{n+k-2}(\Sigma) & =b_{n+k}(\Sigma)+1, \quad b_{n+k-1}(\Sigma)=b_{n+k+1}(\Sigma)+b_{1}(\Sigma) .
\end{aligned}
$$

The proof is given in Section 7 


\section{Proofs of Theorem 4.2 and Theorems A and C}

As noted in the discussion after the statement of Theorem 4.2, Theorems A and C from Section 1 are immediate consequences of Theorem 4.2. Therefore, we will concentrate in this section in proving the latter. We will make throughout this section the same assumptions as in Theorem 4.2 and use here the construction and notation of Section 4.

For a hyperplane $H \in\left(\mathbb{C} P^{N}\right)^{*}$ write $\Sigma_{H}=X \cap H$. For a pencil $\ell \subset\left(\mathbb{C} P^{N}\right)^{*}$ denote by $B_{\ell}=\Sigma_{H_{0}} \cap \Sigma_{H_{1}} \subset X,\left(H_{0}, H_{1} \in \ell\right)$, its base locus. Recall that $p: \tilde{X} \longrightarrow X$ can be viewed as the blowup of $X$ along $B_{\ell}$. Denote by $E \subset \tilde{X}$ the exceptional divisor of this blowup. The restriction $\left.p\right|_{E}: E \longrightarrow B_{\ell}$ is a holomorphic fibration with fiber $\mathbb{C} P^{1}$. Denote the homology class of this fiber by $F \in H_{2}(\tilde{X} ; \mathbb{Z})$. Since $\operatorname{dim}_{\mathbb{R}} B_{\ell}=2 n-4$, the map induced by inclusion $H_{2}\left(X \backslash B_{\ell} ; \mathbb{Z}\right) \longrightarrow H_{2}(X ; \mathbb{Z})$ is an isomorphism, hence we obtain an obvious injection $j:$ $H_{2}(X ; \mathbb{Z}) \longrightarrow H_{2}(\tilde{X} ; \mathbb{Z})$. The second homology of $\tilde{X}$ is then given by

$$
H_{2}(\tilde{X} ; \mathbb{Z})=j\left(H_{2}(X ; \mathbb{Z})\right) \oplus \mathbb{Z} F
$$

The $(2 n-2)$ th homology of $\tilde{X}$ fits into the following exact sequence:

$$
0 \longrightarrow \mathbb{Z}[E] \longrightarrow H_{2 n-2}(\tilde{X} ; \mathbb{Z}) \stackrel{p_{*}}{\longrightarrow} H_{2 n-2}(X ; \mathbb{Z}) \longrightarrow 0
$$

where the first map is induced by the inclusion. We obviously have $p_{*} \circ j=\mathrm{id}$. Denote by $\tilde{\Sigma} \subset \tilde{X}$ the proper transform of $\Sigma$ (with respect to $p$ ) in $\tilde{X}$. The intersection pairing between $H_{2 n-2}$ and $H_{2}$ in $\tilde{X}$ is related to the one in $X$ as follows:

$$
\begin{aligned}
& V \cdot j(A)=p_{*}(V) \cdot A, \quad \forall V \in H_{2 n-2}(\tilde{X} ; \mathbb{Z}), \quad A \in H_{2}(X ; \mathbb{Z}), \\
& {[\tilde{\Sigma}] \cdot F=1, \quad[E] \cdot F=-1, \quad[E] \cdot j(A)=0 \quad \forall A \in H_{2}(X ; \mathbb{Z}) .}
\end{aligned}
$$

Consider now the fibration $\pi_{\ell}: \tilde{X} \longrightarrow \ell$. The fiber over $H_{0} \in \ell$ is precisely $\Sigma=\Sigma_{H_{0}}$. It follows from (9) that the set of classes $H_{2}^{\pi_{\ell}}$ that represent sections of $\pi$ satisfies:

$$
H_{2}^{\pi_{\ell}} \subset\{j(A)+d F \mid[\Sigma] \cdot A=1-d\}
$$

Denote by $J_{0}$ the standard complex structure of $X$ (coming from the structure of $X$ as an algebraic manifold). Denote by $\mathcal{R}(X) \subset H_{2}(X ; \mathbb{Z})$ the positive cone generated by 
classes that represent $J_{0}$-holomorphic rational curves in $X$, that is,

$$
\mathcal{R}(X)=\left\{\sum a_{i}\left[C_{i}\right] \mid a_{i} \in \mathbb{Z}_{\geq 0}, C_{i} \subset X \text { is a rational } J_{0} \text {-holomorphic curve }\right\} .
$$

Lemma 5.1. Let $\tilde{A}=j(A)+d F \in H_{2}^{\pi_{\ell}}$, with $A \in H_{2}(X ; \mathbb{Z}), d \in \mathbb{Z}$. If $\mathcal{S}(\tilde{A}) \neq 0$ then $A \in \mathcal{R}(X)$ and $d \leq 1$, with equality if and only if $A=0$.

Proof. Denote by $\widetilde{J}_{0}$ the standard complex structure on $\tilde{X} \subset X \times \ell$, namely the complex structure induced from the standard complex structure $J_{0} \oplus i$ on $X \times \ell$. Let $\widetilde{J}_{n}$ be a sequence of regular almost complex structures on $\tilde{X}$ with $\widetilde{J}_{n} \longrightarrow \widetilde{J}_{0}$. Since $\mathcal{S}\left(\tilde{A}, \widetilde{J}_{n}\right) \neq 0$, there exist $\widetilde{J}_{n}$-holomorphic sections $u_{n} \in \mathcal{M}^{\mathfrak{s}}\left(\tilde{A}, \widetilde{J}_{n}\right)$. After passing to the limit $n \longrightarrow \infty$, we obtain by Gromov compactness theorem a (possibly reducible) $\widetilde{J}_{0}$-holomorphic curve $D \subset \tilde{X}$ in the class $\tilde{A}$. As $p: \tilde{X} \longrightarrow X$ is $\left(\widetilde{J}_{0}, J_{0}\right)$-holomorphic it follows that $p(D)$ is a $J_{0}$ holomorphic rational curve, hence $A=p_{*}([D]) \in \mathcal{R}(X)$.

Next, recall that $[\Sigma] \cdot A=1-d$. But $\Sigma \subset X$ is ample, hence $[\Sigma] \cdot A=1-d \geq 0$ with equality if and only if $A=0$.

The next lemma shows that when $d<1$ the sections in the class $\tilde{A}$ do not contribute to the Seidel element in (7).

Lemma 5.2. Let $\tilde{A}=j(A)+d F \in H_{2}^{\pi_{\ell}}$ with $A \in H_{2}(X ; \mathbb{Z})$ and $d<1$. Then $c_{1}^{v}(\tilde{A})>0$. In particular, in view of (5), $\tilde{A}$ does not contribute to $S\left(\pi_{\ell}\right)$.

Proof. Denote by $c_{1}^{\tilde{X}}$ the first Chern class of (the tangent bundle of) $\tilde{X}$ and by $c_{1}^{X}$ that of $X$. Since $\tilde{X}$ is the blowup of $X$ along $B_{\ell}$, the relation between these Chern classes is given by

$$
c_{1}^{\tilde{X}}=p^{*} c_{1}^{X}-\operatorname{PD}([E]),
$$

where $\operatorname{PD}([E]) \in H^{2}(\tilde{X})$ stands for the Poincaré dual of $[E]$. (See, e.g., [26].)

Denote by $c_{1}^{\ell}$ the first Chern class of $\ell \cong \mathbb{C} P^{1}$. Since $\tilde{A}$ represents sections of $\pi_{\ell}$ we have

$$
c_{1}^{v}(\tilde{A})=c_{1}^{\tilde{X}}(\tilde{A})-\pi_{\ell}^{*}\left(c_{1}^{\ell}\right)(\tilde{A})=c_{1}^{\tilde{X}}(\tilde{A})-2 .
$$

Together with (11) and (9) this implies:

$$
C_{1}^{v}(\tilde{A})=p^{*}\left(c_{1}^{X}\right)(\tilde{A})-[E] \cdot \tilde{A}-2=c_{1}^{X}(A)+d-2 .
$$


By Lemma 5.1, $A \in \mathcal{R}(X) \subset H_{2}^{S}(X)$, hence by Remark 4.3, we have

$$
c_{1}^{X}(A)=\frac{n+k+2}{2} h(A)=\frac{n+k+2}{2}([\Sigma] \cdot A)=\frac{n+k+2}{2}(1-d) .
$$

Together with (12) we obtain

$$
c_{1}^{v}(\tilde{A})=\frac{n+k}{2}(1-d)-1 \geq \frac{n+k}{2}-1>0,
$$

because $d<1$ and $n \geq 2$.

We now turn to the case $\tilde{A}=F$. Let $b \in B_{\ell}$. Define

$$
\tilde{u}_{b}: \ell \longrightarrow \tilde{X}, \quad \tilde{u}_{b}(z)=(b, z)
$$

It is easy to see that $\tilde{u}_{b}$ is a $\widetilde{J}_{0}$-holomorphic section of $\pi_{\ell}$ representing the class $F$.

Lemma 5.3. The sections $\tilde{u}_{b}$ for $b \in B_{\ell}$ are the only $\widetilde{J}_{0}$-holomorphic sections in the class $F$, hence $\mathcal{M}^{\mathfrak{s}}\left(F, \widetilde{J}_{0}\right)=\left\{\tilde{u}_{b} \mid b \in B_{\ell}\right\}$. The evaluation map is given by

$$
e v_{\widetilde{J}_{0}, z_{0}}\left(\tilde{u}_{b}\right)=b \in \Sigma
$$

and is an orientation preserving diffeomorphism between $\mathcal{M}^{\mathfrak{s}}\left(F, \widetilde{J}_{0}\right)$ and the base locus $B_{\ell}$.

Proof. Let $\tilde{u}: \ell \longrightarrow \tilde{X}$ be a $\widetilde{J}_{0}$-holomorphic section in the class $F$. Write $\tilde{u}(z)=(v(z), z) \in$ $X \times \ell$. Due to our choice of $\widetilde{J}_{0}, v$ is a $J_{0}$-holomorphic map. Since $p_{*}(F)=0$ the map $v=$ $p \circ u: \ell \longrightarrow X$ must be constant, say $v(z) \equiv b, b \in X$. But $v(z) \in \Sigma_{z}$ for every $z \in \ell$. It follows that $b \in \cap_{z \in \ell} \Sigma_{z}=B_{\ell}$. The rest of the statements in the lemma are immediate.

We are now ready for the following.

Proof of Theorem 4.2. In view of (10) and Lemmas 5.1, 5.2, the only class that contributes to the Seidel element $S\left(\pi_{\ell}\right)$ is $F$, hence:

$$
S\left(\pi_{\ell}\right)=\mathcal{S}(F) \in \mathrm{QH}^{2}(\Sigma ; \Lambda) .
$$

(We take $F$ to be the reference class of sections and note that $c_{1}^{v}(F)=-1$.) 
In order to evaluate $\mathcal{S}(F)$, we need to compute $\mathcal{S}(F, \tilde{J})$ for a regular $\tilde{J}$. We first claim that there exists a neighborhood $\mathcal{U}$ of $\widetilde{J}_{0}$ inside $\tilde{\mathcal{J}}\left(\pi_{\ell}, \tilde{\Omega}\right)$ such that for every $\tilde{J} \in \mathcal{U}$ the space $\mathcal{M}^{\mathfrak{s}}(F, \tilde{J})$ is compact.

To see this, first note that $\tilde{\Omega}$ is a genuine symplectic form on $\tilde{X}$ and that $\widetilde{J}_{0}$ is tamed by $\tilde{\Omega}$ (i.e., $\Omega\left(v, \widetilde{J}_{0} v\right)>0$ for all nonzero vectors $v \in T \tilde{X}$ be they vertical or not). Hence there is a neighborhood $\mathcal{U}$ of $\widetilde{J}_{0}$ in $\tilde{\mathcal{J}}\left(\pi_{\ell}, \tilde{\Omega}\right)$ such that every $\tilde{J} \in \mathcal{U}$ is tamed by $\tilde{\Omega}$. Next note that $\tilde{\Omega}$ defines an integral (modulo torsion) cohomology class $[\tilde{\Omega}] \in H^{2}(\tilde{X})_{\text {free }}$ and that $\tilde{\Omega}(F)=1$ (see Section 4). It follows that $F$ is a class of minimal positive area for $\tilde{\Sigma}$. Therefore, for $\tilde{J}$ tamed by $\tilde{\Omega}$, a sequence of $\tilde{J}$-holomorphic rational curves in the class $F$ cannot develop bubbles. By Gromov compactness $\mathcal{M}^{\mathfrak{s}}(F, \tilde{J})$ is compact.

Next, we claim that $\widetilde{J}_{0}$ is a regular almost complex structure in the sense of the general theory of pseudo-holomorphic curves (see [37, Chapter 3]). To see this, recall the following regularity criterion (see [37, Lemma 3.3.1]): let $(M, \omega)$ be a symplectic manifold and $J$ an integrable almost complex structure. Then $J$ is regular for a $J$-holomorphic curve $u: \mathbb{C} P^{1} \longrightarrow M$ if every summand of the holomorphic bundle $u^{*} T M \rightarrow \mathbb{C} P^{1}$ (in its splitting to a direct sum of line bundles) has Chern number $\geq-1$. Applying this to our case, a simple computation shows that for every $\tilde{u}_{b} \in \mathcal{M}^{\mathfrak{s}}\left(F, \widetilde{J}_{0}\right)$, we have

$$
\tilde{u}_{b}^{*} T \tilde{X}=\mathcal{O}_{\ell}(2) \oplus \mathcal{O}_{\ell}^{\oplus(n-2)} \oplus \mathcal{O}_{\ell}(-1)
$$

hence $\widetilde{J}_{0}$ is regular for all $\tilde{u} \in \mathcal{M}^{\mathfrak{s}}\left(F, \widetilde{J}_{0}\right)$.

Pick a regular almost complex structure $\tilde{J} \in \tilde{\mathcal{J}}_{\text {reg }}(\pi, \tilde{\Omega}) \cap \mathcal{U}$ which is close enough to $\widetilde{J}_{0}$. By the standard theory of pseudo-holomorphic curves [37] the evaluation maps $e v_{\tilde{J}, z_{0}}$ and $e v_{\widetilde{J}_{0}, z_{0}}$ are cobordant, hence give rise to cobordant pseudo-cycles. Moreover by what we have seen before this cobordism can be assumed to be compact (and the pseudo-cycles are in fact cycles). It follows that the homology class $\left(e v_{\tilde{J}, z_{0}}\right)_{*}\left[\mathcal{M}^{\mathfrak{s}}(F, \tilde{J})\right]$ equals to $\left(e v_{\widetilde{J}_{0}, z_{0}}\right)_{*}\left[\mathcal{M}^{\mathfrak{s}}\left(F, \widetilde{J}_{0}\right)\right]=\left[B_{\ell}\right]$. Putting everything together we obtain:

$$
S\left(\pi_{\ell}\right)=\mathcal{S}(F, \tilde{J})=\operatorname{PD}\left(\left[B_{\ell}\right]\right)=\left[\omega_{\Sigma}\right]
$$

\section{Subcriticality and Projective Defect}

Here, we discuss symplectic and topological aspects of manifolds $X$ with small dual that have to do with the structure of the (affine) Stein manifold obtained after removing from $X$ a hyperplane section. Some of the results of this section should be known to experts, but we could not find them in explicit form in the literature. We therefore state 
the results and occasionally refer the reader to proofs that can be found in the expanded version of this paper [14].

Let $Y \subset \mathbb{C}^{N}$ be a Stein manifold. The study of Morse theory on Stein manifolds was initiated in the classical paper [1] of Andreotti and Frankel and in its sequel [2]. Further aspects of Morse theory as well as symplectic topology on Stein manifolds were studied by various authors [10, 22-24]. In this context, it is important to remark that by a result of Eliashberg-Gromov [23, 24], Stein manifolds $Y$ admit a canonical symplectic structure $\hat{\omega}_{Y}$ (see also [10]).

A function $\varphi: Y \longrightarrow \mathbb{R}$ is called plurisubharmonic (p.s.h. in short) if the form $\Omega=-d d^{\mathbb{C}} \varphi$ is a Kähler form on Y. Here, $d^{\mathbb{C}} \varphi=d \varphi \circ J$, where $J$ is the complex structure of $Y$. A plurisubharmonic function $\varphi: Y \rightarrow \mathbb{R}$ is called exhausting if it is proper and bounded from below. For a plurisubharmonic Morse function $\varphi: Y \longrightarrow \mathbb{R}$ denote

$$
\operatorname{ind}_{\max }(\varphi)=\max \left\{\operatorname{ind}_{z}(\varphi) \mid z \in \operatorname{Crit}(\varphi)\right\},
$$

where $\operatorname{ind}_{z}(\varphi)$ is the Morse index of the critical point $z \in \operatorname{Crit}(\varphi)$. A fundamental property of plurisubharmonic Morse functions $\varphi$ is that $\operatorname{ind}_{\max }(\varphi) \leq \operatorname{dim}_{\mathbb{C}}(Y)$. (Various proofs of this can be found in, e.g., [1, 22-24].)

A Stein manifold $Y$ is called subcritical if it admits an exhausting plurisubharmonic Morse function $\varphi: Y \longrightarrow \mathbb{R}$ with $\operatorname{ind}_{\max }(\varphi)<\operatorname{dim}_{\mathbb{C}}(Y)$ for every $z \in \operatorname{Crit}(\varphi)$. Otherwise, we call $Y$ critical. The subcriticality index $\operatorname{ind}(Y)$ of a Stein manifold $Y$ is defined by

$\operatorname{ind}(Y):=\min \left\{\operatorname{ind}_{\max }(\varphi) \mid \varphi: Y \longrightarrow \mathbb{R}\right.$ is a p.s.h. exhausting Morse function $\}$

Thus, we have $0 \leq \operatorname{ind}(Y) \leq \operatorname{dim}_{\mathbb{C}}(Y)$, and $Y$ is subcritical iff $\operatorname{ind}(Y)<\operatorname{dim}_{\mathbb{C}}(Y)$.

Subcritical Stein manifolds $Y$ have special symplectic properties (when endowed with their canonical symplectic structure $\left.\hat{\omega}_{Y}\right)$. For example, every compact subset $A \subset Y$ is Hamiltonianly displaceable. In particular, whenever well defined, the Floer homology $\operatorname{HF}\left(L_{1}, L_{2}\right)$ of every pair of compact Lagrangian submanifolds $L_{1}, L_{2} \subset Y$ vanishes. This in turn implies strong topological restrictions on the Lagrangian submanifolds of $Y$ (see, e.g., [10]). Such considerations will play an important role in Section 8.

The main result in this context is the following. 
Theorem 6.1. Let $X \subset \mathbb{C} P^{N}$ be a projective manifold with small dual and let $\Sigma \subset X$ be a smooth hyperplane section of $X$. Then the Stein manifold $X \backslash \Sigma$ is subcritical. In fact,

$$
\operatorname{ind}(X \backslash \Sigma) \leq \operatorname{dim}_{\mathbb{C}}(X)-\operatorname{def}(X)
$$

See Theorem 8.5 for a partial converse to this theorem.

Theorem 6.1 can easily be proved using the the theory developed in [2]. An alternative proof can be found in the expanded version of this paper [14].

Using standard arguments one gets from Theorem 6.1, the following version of the Lefschetz hyperplane theorem for manifolds with small dual.

Corollary 6.2. Let $X \subset \mathbb{C} P^{N}$ be an algebraic manifold with $\operatorname{dim}_{\mathbb{C}} X=n$ and $\operatorname{def}(X)=k$ and let $\Sigma \subset X$ be a smooth hyperplane section. Denote by $i: \Sigma \longrightarrow X$ the inclusion. The induced maps $i_{*}: H_{j}(\Sigma ; \mathbb{Z}) \longrightarrow H_{j}(X ; \mathbb{Z})$ and $i_{*}: \pi_{j}(\Sigma, *) \longrightarrow \pi_{j}(X, *)$ are

(1) Isomorphisms for $j<n+k-1$.

(2) Surjective for $j=n+k-1$.

Similarly, the restriction map $i^{*}: H^{j}(X ; \mathbb{Z}) \longrightarrow H^{j}(\Sigma ; \mathbb{Z})$ is an isomorphism for every $j<n+k-1$ and injective for $j=n+k-1$.

Corollary 6.2 was previously known and proved in [33] by other methods. It can also be derived from the theory in the earlier paper [2].

Another consequence is the following refinement of the hard Lefschetz theorem.

Corollary 6.3. Let $X \subset \mathbb{C} P^{N}$ be as in Corollary 6.2. Denote by $\omega$ the Kähler form on $X$ induced from the standard Kähler form of $\mathbb{C} P^{N}$. Then the map

$$
L: H^{j}(X ; \mathbb{R}) \longrightarrow H^{j+2}(X ; \mathbb{R}), \quad L(a)=a \cup[\omega]
$$

is an isomorphism for every $n-k-1 \leq j \leq n+k-1$.

Proof. This follows from Corollary 6.2 together with the Hard Lefschetz theorem applied both to $\Sigma$ and $X$. 


\section{Proof of Corollary 4.4}

The quantum cohomology of $\Sigma$ can be written additively (as a vector space) as

$$
\mathrm{QH}^{j}(\Sigma ; \Lambda) \cong \bigoplus_{l \in \mathbb{Z}} H^{j+2 C_{\Sigma} l}(\Sigma)
$$

By Theorem 4.2, $\left[\omega_{\Sigma}\right] \in \mathrm{QH}^{2}(\Sigma ; \Lambda)$ is invertible with respect to the quantum product $*$, hence the map

$$
(-) *\left[\omega_{\Sigma}\right]: \mathrm{OH}^{j}(\Sigma ; \Lambda) \longrightarrow \mathrm{OH}^{j+2}(\Sigma ; \Lambda), \quad a \longmapsto a *\left[\omega_{\Sigma}\right]
$$

is an isomorphism for every $j \in \mathbb{Z}$. The statement about $\tilde{b}_{j}(\Sigma)$ follows immediately.

We now turn to the proof of the statement about $\tilde{b}_{j}(X)$. First recall that $2 C_{\Sigma}=n+$ $k$ and $2 C_{X}=n+k+2$. We will show now that for every $0 \leq j \leq n+k+1$ we have $\tilde{b}_{j}(X)=$ $\tilde{b}_{j+2}(X)$.

Step 1. Assume $j \leq n+k-4$. By Corollary $6.2, b_{j}(\Sigma)=b_{j}(X)$ and $b_{j+2}(\Sigma)=$ $b_{j+2}(X)$. We claim that

$$
b_{j+n+k}(\Sigma)=b_{j+n+k+2}(X), \quad b_{j+n+k+2}(\Sigma)=b_{j+n+k+4}(X) .
$$

Indeed, by Corollary 6.2, $b_{n-j-k-2}(\Sigma)=b_{n-j-k-2}(X)$, hence the first equation in (13) follows from Poincaré duality for $\Sigma$ and $X$. The proof of the second equality is similar.

It follows that

$$
\begin{aligned}
\tilde{b}_{j}(X) & =b_{j}(X)+b_{j+n+k+2}(X)=b_{j}(\Sigma)+b_{j+n+k}(\Sigma)=\tilde{b}_{j}(\Sigma) \\
& =\tilde{b}_{j+2}(\Sigma)=b_{j+2}(\Sigma)+b_{j+n+k+2}(\Sigma)=b_{j+2}(X)+b_{j+n+k+4}(X)=\tilde{b}_{j+2}(X) .
\end{aligned}
$$

Step 2. Assume $n+k-3 \leq j \leq n+k-1$. In this case, we have $\tilde{b}_{j}(X)=b_{j}(X)$ and $\tilde{b}_{j+2}(X)=b_{j+2}(X)$ and the equality between the two follows from Corollary 6.3.

Step 3. Assume $j=n+k$. We have to prove that $b_{n+k}(X)=b_{0}(X)+b_{n+k+2}(X)$. By Poincaré duality, this is equivalent to showing that $b_{n-k}(X)=b_{0}(X)+b_{n-k-2}(X)$. The last equality is, by Corollary 6.2, equivalent to $b_{n-k}(\Sigma)=b_{0}(\Sigma)+b_{n-k-2}(\Sigma)$. Applying Poincaré duality on $\Sigma$ the latter becomes equivalent to $b_{n+k-2}(\Sigma)=b_{0}(\Sigma)+b_{n+k}(\Sigma)$. But this has already been proved since $b_{n+k-2}(\Sigma)=\tilde{b}_{n+k-2}(\Sigma)=\tilde{b}_{n+k}(\Sigma)=b_{0}(\Sigma)+b_{n+k}(\Sigma)$.

Step 4. Assume $j=n+k+1$. The proof in this case is very similar to the case $j=n+k$. We omit the details. 


\section{Further Results}

As we have seen above, the algebraic geometry of manifolds with small dual is intimately connected with their symplectic topology. Here, we add another ingredient which has to do with Lagrangian submanifolds. Below, we will use the following notation. For an algebraic manifold $X \subset \mathbb{C} P^{N}$ and an algebraic submanifold $\Sigma \subset X$ we denote by $\omega_{X}$ and $\omega_{\Sigma}$ the restrictions of the standard Kähler form of $\mathbb{C} P^{N}$ to $X$ and to $\Sigma$, respectively.

The following theorem follows easily by combining results from [2] with the fact that vanishing cycles can be represented by Lagrangian spheres [3, 17, 43]. (See also [6], Theorem K, [7], Theorem 2.1.)

Theorem 8.1. Let $X \subset \mathbb{C} P^{N}$ be an algebraic manifold and $\Sigma \subset X$ a hyperplane section. If $\operatorname{def}(X)=0$, then $\left(\Sigma, \omega_{\Sigma}\right)$ contains a (embedded) Lagrangian sphere.

Thus we can detect manifolds with small dual (i.e., def $>0$ ) by methods of symplectic topology, for example, by showing that their hyperplane sections do not contain Lagrangian spheres.

In some situations we also have the converse to Theorem 8.1.

Theorem 8.2. Let $\Sigma \subset X \subset \mathbb{C} P^{N}$ be as in Theorem 4.2 and assume in addition that $\operatorname{dim}_{\mathbb{C}}(\Sigma) \geq 3$. Then the symplectic manifold $\left(\Sigma, \omega_{\Sigma}\right)$ contains no Lagrangian spheres.

Remark 8.3. Note that from the results of [2] it follows that the (homological) subgroup of vanishing cycles $V_{n-1} \subset H_{n-1}(\Sigma)$ of $\Sigma$ is trivial (here, $n-1=\operatorname{dim}_{\mathbb{C}} \Sigma$ ). Theorem 8.2, asserting that $\Sigma$ has no Lagrangian spheres, is however stronger. Indeed, it is not known whether or not every Lagrangian sphere comes from a vanishing cycle. Moreover in some cases Lagrangian spheres do exists but are null-homologous. (Put differently, in general it is not possible to use purely topological methods to prove nonexistence of Lagrangian spheres.)

Proof of Theorem 8.2. Suppose by contradiction that $L \subset\left(\Sigma, \omega_{\Sigma}\right)$ is a Lagrangian sphere. We will use now the theory of Lagrangian Floer cohomology for in order to arrive at a contradiction. More specifically, we will use here a particular case of the general theory that works for so called monotone Lagrangian submanifolds. We will take $\mathbb{Z}_{2}$ as the ground ring and work with the Floer self-cohomology of $L$, denoted $\operatorname{HF}(L, L)$, with coefficients in the Novikov ring $\Lambda_{\mathbb{Z}_{2}}=\mathbb{Z}_{2}\left[q, q^{-1}\right]$. This ring is graded so that the variable 
$q$ has degree $\operatorname{deg}(q)=N_{L}$, where $N_{L}$ is the minimal Maslov number of $L$. We refer the reader to $[12,13,39,40]$ for the foundations of this theory.

Since $L$ is simply connected, the assumptions on $\Sigma$ and $X$ imply that $L \subset \Sigma$ is a monotone Lagrangian submanifold and its minimal Maslov number is $N_{L}=2 C_{\Sigma}=n+k$. (Here, as in Theorem $4.2, k=\operatorname{def}(X) \geq 1$.) Under these circumstances, it is well known that the Floer self-cohomology of $L, \operatorname{HF}(L, L)$ is well defined and moreover we have an isomorphism of graded $\Lambda_{\mathbb{Z}_{2}}$-modules:

$$
\operatorname{HF}^{*}(L, L) \cong\left(H^{\bullet}\left(L ; \mathbb{Z}_{2}\right) \otimes \Lambda_{\mathbb{Z}_{2}}\right)^{*}
$$

Since $L$ is a sphere of $\operatorname{dimension}_{\operatorname{dim}_{\mathbb{R}}}(L) \geq 3$ this implies that

$$
\operatorname{HF}^{0}(L, L) \cong \mathbb{Z}_{2}, \quad \operatorname{HF}^{2}(L, L) \cong H^{2}\left(L ; \mathbb{Z}_{2}\right)=0
$$

Denote by $\mathrm{OH}\left(\Sigma ; \Lambda_{\mathbb{Z}_{2}}\right)$ the modulo-2 reduction of $\mathrm{OH}(\Sigma ; \Lambda)$ (obtained by reducing the ground ring $\mathbb{Z}$ to $\left.\mathbb{Z}_{2}\right)$. By Theorem $4.2,\left[\omega_{\Sigma}\right] \in \mathrm{OH}^{2}(\Sigma ; \Lambda)$ is an invertible element, hence its modulo-2 reduction, say $\alpha \in \mathrm{OH}^{2}\left(\Sigma ; \Lambda_{\mathbb{Z}_{2}}\right)$ is invertible too.

We now appeal to the quantum module structure of $\operatorname{HF}(L, L)$ introduced in [11-13]. By this construction, $\operatorname{HF}(L, L)$ has a structure of a graded module over the ring $\mathrm{QH}\left(\Sigma ; \Lambda_{\mathbb{Z}_{2}}\right)$, where the latter is endowed with the quantum product. We denote the module action of $\mathrm{OH}^{*}\left(\Sigma ; \Lambda_{\mathbb{Z}_{2}}\right)$ on $\operatorname{HF}^{*}(L, L)$ by

$$
\mathrm{QH}^{i}\left(\Sigma ; \Lambda_{\mathbb{Z}_{2}}\right) \otimes_{\Lambda_{\mathbb{Z}_{2}}} \mathrm{HF}^{j}(L, L) \longrightarrow \mathrm{HF}^{i+j}(L, L), \quad a \otimes X \longmapsto a \circledast x, i, j \in \mathbb{Z} .
$$

Since $\alpha \in \mathrm{OH}^{2}\left(\Sigma ; \Lambda_{\mathbb{Z}_{2}}\right), \quad \alpha$ induces an isomorphism $\alpha \circledast(-): \operatorname{HF}^{*}(L, L) \longrightarrow$ $\operatorname{HF}^{*+2}(L, L)$. This, however, is impossible (e.g., for $*=0$ ) in view of (14). Contradiction.

Corollary 8.4. Let $\Sigma$ be an algebraic manifold with $\operatorname{dim}_{\mathbb{C}}(\Sigma) \geq 3$ and $b_{2}(\Sigma)=1$. Suppose that $\Sigma$ can be realized as a hyperplane section of a projective manifold $X \subset \mathbb{C} P^{N}$ with small dual. Then in any other realization of $\Sigma$ as a hyperplane section of a projective manifold $X^{\prime} \subset \mathbb{C} P^{N^{\prime}}$, we have $\operatorname{def}\left(X^{\prime}\right)>0$. In fact, $\operatorname{def}\left(X^{\prime}\right)=\operatorname{def}(X)$.

Proof. Let $\omega_{\Sigma}$ be the restriction to $\Sigma$ (via $\Sigma \subset X \subset \mathbb{C} P^{N}$ ) of the standard symplectic structure of $\mathbb{C} P^{N}$. Similarly, let $\omega_{\Sigma}^{\prime}$ the restriction to $\Sigma$ (via $\Sigma \subset X^{\prime} \subset \mathbb{C} P^{N^{\prime}}$ ) of the standard symplectic structure of $\mathbb{C} P^{N^{\prime}}$. 
Since $b_{2}(\Sigma)=1$, it follows from Lefschetz theorem that $b_{2}(X)=1$. Thus, $X$ satisfies the conditions of Theorem 4.2 (see the discussion after Theorem 4.2). By Theorem 8.2, the symplectic manifold $\left(\Sigma, \omega_{\Sigma}\right)$ does not contain Lagrangian spheres.

Since $b_{2}(\Sigma)=1$ the cohomology classes $\left[\omega_{\Sigma}\right]$ and $\left[\omega_{\Sigma}^{\prime}\right]$ are proportional, so there is a constant $c$ such that $\left[\omega_{\Sigma}^{\prime}\right]=c\left[\omega_{\Sigma}\right]$. Clearly, we have $c>0$ (to see this, take an algebraic curve $D \subset \Sigma$ and note that both $\int_{D} \omega_{\Sigma}$ and $\int_{D} \omega_{\Sigma}^{\prime}$ must be positive since both $\omega_{\Sigma}$ and $\omega_{\Sigma}^{\prime}$ are Kähler forms with respect to the complex structure of $\Sigma$ ). We claim that the symplectic structures $\omega_{\Sigma}^{\prime}$ and $c \omega_{\Sigma}$ are diffeomorphic, that is, there exists a diffeomorphism $\varphi: \Sigma \longrightarrow \Sigma$ such that $\varphi^{*} \omega_{\Sigma}^{\prime}=c \omega_{\Sigma}$. Indeed this follows from Moser argument [36] since all the forms in the family $\left\{(1-t) c \omega_{\Sigma}+t \omega_{\Sigma}^{\prime}\right\}_{t \in[0,1]}$ are symplectic (since $c \omega_{\Sigma}$ and $\omega_{\Sigma}^{\prime}$ are both Kähler with respect to the same complex structure) and all lie in the same cohomology class.

Since $\left(\Sigma, c \omega_{\Sigma}\right)$ has no Lagrangian spheres the same holds for $\left(\Sigma, \omega_{\Sigma}^{\prime}\right)$ too. By Theorem 8.1, we have $\operatorname{def}\left(X^{\prime}\right)>0$.

That $\operatorname{def}\left(X^{\prime}\right)=\operatorname{def}(X)$ follows immediately from the fact that for manifolds with positive defect the minimal Chern number $C_{\Sigma}$ of a hyperplane section $\Sigma$ is determined by the defect. More specifically, we have (see Section 2)

$$
\frac{n+\operatorname{def}(X)}{2}=C_{\Sigma}=\frac{n+\operatorname{def}\left(X^{\prime}\right)}{2},
$$

where $n=\operatorname{dim}_{\mathbb{C}}(X)$.

Theorem 6.1 says that the complement of a hyperplane section $X \backslash \Sigma$ of an algebraic manifold $X \subset \mathbb{C} P^{N}$ with small dual is subcritical. Here is a partial converse:

Theorem 8.5. Let $X \subset \mathbb{C} P^{N}$ be an algebraic manifold with $n=\operatorname{dim}_{\mathbb{C}}(X) \geq 3$ and let $\Sigma \subset X$ be a hyperplane section. Assume that $\left(\Sigma, \omega_{\Sigma}\right)$ is spherically monotone with $C_{\Sigma} \geq 2$ and that $2 C_{\Sigma}$ does not divide $n$. If $X \backslash \Sigma$ is subcritical, then $\operatorname{def}(X)>0$.

Note that the spherical monotonicity of $\left(\Sigma, \omega_{\Sigma}\right)$ is automatically satisfied, for example, when $\Sigma$ is Fano and $b_{2}(\Sigma)=1$.

Proof. Suppose by contradiction that $\operatorname{def}(X)=0$. By Theorem $8.1\left(\Sigma, \omega_{\Sigma}\right)$ has a Lagrangian sphere, say $L \subset \Sigma$. Note that since $\Sigma$ is spherically monotone, the Lagrangian $L \subset \Sigma$ is monotone too and since $L$ is simply connected its minimal Maslov number is $N_{L}=2 C_{\Sigma}$. 
Put $W=X \backslash \Sigma$ endowed with the symplectic form $\omega_{W}$ induced from $X$ (which in turn is induced from $\mathbb{C} P^{N}$ ). We now appeal to the Lagrangian circle bundle construction introduced in $[8,9]$. We briefly recall the construction. Pick a tubular neighborhood $\mathcal{U}$ of $\Sigma$ in $X$ whose boundary $\partial \mathcal{U}$ is a circle bundle over $\Sigma$. Denote this circle bundle by $\pi: \partial \mathcal{U} \rightarrow \Sigma$. Then $\Gamma_{L}=\pi^{-1}(L)$ is the total space of a circle bundle over $L$, embedded inside $W$. By the results of [8], for a careful choice of $\mathcal{U}$ the submanifold $\Gamma_{L}$ is Lagrangian in $W$. Moreover, since $L$ is monotone $\Gamma_{L}$ is monotone too and has the same minimal Maslov number: $N_{\Gamma_{L}}=N_{L}=2 C_{\Sigma}$. (See [8] for more details.)

Denote by $\left(\hat{W}, \hat{\omega}_{W}\right)$ the symplectic completion of the symplectic Stein manifold $\left(W, \omega_{W}\right)$ (see $[8,10]$ for the details). By the results of [10], $\Gamma_{L}$ is Hamiltonianly displaceable (i.e., there exists a compactly supported Hamiltonian diffeomorphism $h:\left(\hat{W}, \hat{\omega}_{W}\right) \longrightarrow$ $\left(\hat{W}, \hat{\omega}_{W}\right)$ such that $\left.h\left(\Gamma_{L}\right) \cap \Gamma_{L}=\emptyset\right)$. In particular, $\operatorname{HF}\left(\Gamma_{L}, \Gamma_{L}\right)=0$.

One can arrive now at a contradiction by using an alternative method to compute $\mathrm{HF}\left(\Gamma_{L}, \Gamma_{L}\right)$ such as the Oh spectral sequence [8, 40]. (This is a spectral sequence whose initial page is the singular homology of $\Gamma_{L}$ and which converges to $\operatorname{HF}\left(\Gamma_{L}, \Gamma_{L}\right)$, which is 0 in our case.) We will not perform this computation here since the relevant part of it has already been done in [8], hence we will use the latter.

Here are the details. We first claim that the bundle $\left.\pi\right|_{\Gamma_{L}}: \Gamma_{L} \rightarrow L$ is topologically trivial. To see this denote by $N_{\Sigma / X}$ the normal bundle of $\Sigma$ in $X$, viewed as a complex line bundle. Note that $\Gamma_{L} \rightarrow L$ is just the circle bundle associated to $\left.N_{\Sigma / X}\right|_{L}$. Thus, it is enough to show that $\left.N_{\Sigma / X}\right|_{L}$ is trivial. Denote by $c \in H^{2}(\Sigma ; \mathbb{Z})$ the first Chern class of $N_{\Sigma / X}$ and by $c_{\mathbb{R}}$ its image in $H^{2}(\Sigma ; \mathbb{R})$. Similarly, denote by $\left.c\right|_{L}$ and by $\left.c_{\mathbb{R}}\right|_{L}$ the restrictions of $c$ and $c_{\mathbb{R}}$ to $L$. As $\Sigma \subset X$ is a hyperplane section we have $C_{\mathbb{R}}=\left[\omega_{\Sigma}\right]$. But $L \subset\left(\Sigma, \omega_{\Sigma}\right)$ is Lagrangian hence $\left.c_{\mathbb{R}}\right|_{L}=0$. As $H^{*}(L ; \mathbb{Z})$ has no torsion ( $L$ is a sphere) it follows that $\left.C\right|_{L}=0$ too. Thus, the restriction $\left.N_{\Sigma / X}\right|_{L}$ of $N_{\Sigma / X}$ to $L$ has zero first Chern class. This implies that the line bundle $\left.N_{\Sigma / X}\right|_{L} \rightarrow L$ is trivial (as a smooth complex line bundle). In particular, $\Gamma_{L} \rightarrow L$ is a trivial circle bundle.

Since $\Gamma_{L} \approx L \times S^{1}$, we have $H^{i}\left(\Gamma_{L} ; \mathbb{Z}_{2}\right)=\mathbb{Z}_{2}$ for $i=0,1, n-1, n$ and $H^{i}\left(\Gamma_{L} ; \mathbb{Z}_{2}\right)=0$ for every other $i$. By [8, Proposition 6.A], we have $2 C_{\Sigma} \mid n$. A contradiction. (Note that the conditions $n \geq 3$ and $C_{\Sigma} \geq 2$ in the statement of the theorem are in fact required for [8, Proposition 6.A ] to hold.)

\subsection{Other approaches to proving Corollary B}

Here, we briefly outline an alternative approach to proving Corollary B and possibly Theorem A, based on the subcriticality of $X \backslash \Sigma$ that was established in Theorem 6.1. 
Put $W=X \backslash \Sigma$ and $\omega_{W}$ be the symplectic form on $W$ induced from that of $X$. Let $\mathcal{U}$ be a tubular neighborhood of $\Sigma$ in $X$ as in the proof of Theorem 8.5. The boundary $P=\partial \mathcal{U}$ of $\mathcal{U}$ is a circle bundle $\pi: P \longrightarrow \Sigma$ over $\Sigma$. Consider the embedding

$$
i: P \longrightarrow W \times \Sigma, \quad i(p)=(p, \pi(p))
$$

Denote by $\Gamma_{P}=i(P) \subset W \times \Sigma$ the image of $i$. By the results of [8], one can choose $\mathcal{U}$ in such a way that there exists a positive constant (depending on the precise choice of $\mathcal{U}$ ) such that $i(P)$ is a Lagrangian submanifold of ( $\left.W \times \Sigma, \omega_{W} \oplus-C \omega_{\Sigma}\right)$. (Note the minus sign in front of $\omega_{\Sigma}$.) Moreover, the Lagrangian $\Gamma_{P}$ is monotone and its minimal Maslov number is $N_{P}=2 C_{\Sigma}$, where $C_{\Sigma}$ is the minimal Chern number of $\Sigma$. So, by the results recalled in Section 2, we have $N_{P}=n+k$. Note that $\operatorname{dim}_{\mathbb{R}} \Gamma_{P}=2 n+1$.

As $W$ is subcritical it follows that $\Gamma_{P}$ can be Hamiltonianly displaced in the completion $\left(\hat{W} \times \Sigma, \hat{\omega}_{W} \oplus-c \omega_{\Sigma}\right)$ and therefore

$$
\operatorname{HF}\left(\Gamma_{P}, \Gamma_{P}\right)=0
$$

(See [8] for the details. See also the proof of Theorem 8.5.) Note that in order to use here Floer cohomology with ground coefficient ring $\mathbb{Z}$, we need to have $\Gamma_{P}$ oriented and endowed with a spin structure. In our case, $\Gamma_{P}$ carries a natural orientation and it is easy to see that it has a spin structure (in fact, it is easy to see that $H^{1}\left(P ; \mathbb{Z}_{2}\right)=0$ hence this spin structure is unique).

We now appeal to the Oh spectral sequence $[8,40]$. Recall that this is a spectral sequence whose first page is the singular cohomology of $\Gamma_{P}$ and which converges to the Floer cohomology $\operatorname{HF}\left(\Gamma_{P}, \Gamma_{P}\right)$. A simple computation shows that in our case, due to the fact that $N_{P}=n+k$, this sequence collapses at the second page, and moreover since $\operatorname{HF}\left(\Gamma_{P}, \Gamma_{P}\right)=0$ this second page is 0 everywhere. By analyzing the differentials on the first page we obtain the following exact sequences for every $j \in \mathbb{Z}$ :

$$
H^{j-1+n+k}\left(\Gamma_{P} ; \mathbb{Z}\right) \longrightarrow H^{j}\left(\Gamma_{P} ; \mathbb{Z}\right) \longrightarrow H^{j+1-n-k}\left(\Gamma_{P} ; \mathbb{Z}\right)
$$

This implies many restrictions on the cohomology of $P \approx \Gamma_{P}$, for example, that $H^{j}(P ; \mathbb{Z})=0$ for every $n-k+3 \leq j \leq n+k-2$, that $H^{j}(P ; \mathbb{Z}) \cong H^{j-1+n+k}(P ; \mathbb{Z})$ for every $0 \leq j \leq n-k-2$ and more. We now substitute this information into the Gysin sequences of the bundle $P \longrightarrow \Sigma$ (whose Euler class is just the hyperplane class $h$ corresponding to the embedding $\Sigma \subset \mathbb{C} P^{N}$ ). Combining the calculation via the Gysin sequences together 
with the Lefschetz theorem yields the desired periodicity for the cohomology of $\Sigma$. We omit the details as they are rather straightforward.

One could try to push the above argument further by using the methods of [15] (see, e.g., Section 14 in that paper) in order to prove Theorem A via Lagrangian Floer cohomology. However, this would require an extension of the methods of [15] to coefficients in $\mathbb{Z}$ rather than just $\mathbb{Z}_{2}$.

\section{What Happens in the NonMonotone Case}

Here, we briefly explain what happens in Theorem 4.2 when the condition " $C_{1}^{X}(A)=\lambda h(A)$ for some $\lambda>0^{\prime \prime}$ is not satisfied, e.g., when $\left(\Sigma, \omega_{\Sigma}\right)$ is not spherically monotone (see Definition 3.1).

We will need to change here a bit our coefficient ring for the quantum cohomology since $\left(\Sigma, \omega_{\Sigma}\right)$ is not spherically monotone anymore. Denote by $\mathcal{A}$ the ring of all formal series in the variables $q, T$

$$
P(q, T)=\sum_{i, j} a_{i, j} q^{i} T^{s_{j}}, \quad a_{i, j} \in \mathbb{Z}, s_{j} \in \mathbb{R}
$$

which satisfy that for every $C \in \mathbb{R}$

$$
\#\left\{(i, j) \mid a_{i, j} \neq 0 \text { and } s_{j}>C\right\}<\infty .
$$

This ring is a special case of the more general Novikov ring commonly used in the theory of quantum cohomology. With this ring as coefficients, the definition of the quantum product $*$ on $\operatorname{OH}(\Sigma ; \mathcal{A})$ is very similar to what we have had before. Namely, the powers of the variable $q$ will encode Chern numbers of rational curves involved in the definition of $*$ and the powers of $T$ encode their symplectic areas. See [37] for more details.

We now turn to the Hamiltonian fibration $\pi_{\ell}: \tilde{X} \longrightarrow \ell$. We will use here the construction and notation from Sections 4 and 5. Additionally, denote by $\tilde{i}: \Sigma \longrightarrow \tilde{X}$ the inclusion of the fiber into the total space of the fibration $\pi_{\ell}: \tilde{X} \longrightarrow \ell$. Recall also from Section 5 that we have a canonical injection $j: H_{2}(X ; \mathbb{Z}) \longrightarrow H_{2}(\tilde{X} ; \mathbb{Z})$ which satisfies $j \circ p_{*}=\mathrm{id}$, where $p: \tilde{X} \longrightarrow X$ is the blow down map. Denote by $B_{\ell} \subset X$ the base locus of the pencil $\ell$. With this notation we have

$$
\tilde{i}_{*}(\alpha)=j(\alpha)-\left(\left[B_{\ell}\right] \cdot \alpha\right) F=j(\alpha)-\left\langle\left[\omega_{\Sigma}\right], \alpha\right\rangle F \quad \forall \alpha \in H_{2}(\Sigma ; \mathbb{Z}) .
$$


The symplectic form $\tilde{\Omega}$ satisfies:

$$
\begin{aligned}
{[\tilde{\Omega}] } & =2 p^{*}\left[\omega_{X}\right]-e \quad \text { where } e \in H^{2}(\tilde{X}) \text { is the Poincare dual of } E, \\
\langle[\tilde{\Omega}], j(A)\rangle & =2\left\langle\left[\omega_{X}\right], A\right\rangle \quad \forall A \in H_{2}(X ; \mathbb{Z}), \\
\langle[\tilde{\Omega}], F\rangle & =1 .
\end{aligned}
$$

The Seidel element of the fibration $\pi_{\ell}: \tilde{X} \longrightarrow \ell$ will now be

$$
S\left(\pi_{\ell}\right)=\sum_{\tilde{A} \in H_{2}^{\pi}} \mathcal{S}(\tilde{A} ; \tilde{J}) \otimes q^{\nu(\tilde{A})} T^{\langle[\tilde{\Omega}], \tilde{A}\rangle} \in \mathrm{OH}^{-2 c_{0}\left(\pi_{\ell}\right)}(\Sigma ; \mathcal{A})
$$

Some parts of the proof of Theorem 4.1 go through in this new setting. More specifically, Lemma 5.1 as well as Lemma 5.3 continue to hold (with the same proofs) and it follows that the contribution of the class $F$ to the Seidel element is as before, namely

$$
\mathcal{S}(F)=\left[\omega_{\Sigma}\right]
$$

If we choose as before the reference class of sections to be $F$ then the total degree of the Seidel element $S\left(\pi_{\ell}\right)$ continues to be 2 .

In contrast to the above, Lemma 5.2 does not hold anymore since we might have holomorphic sections in the class $\tilde{A}=j(A)+d F$ with $d \leq 0$. (We will see in Section 9.1 an example in which this is indeed the case.) Nevertheless, we can still obtain some information on $S\left(\pi_{\ell}\right)$ beyond (18). Let $d \in \mathbb{Z}$ and put $\tilde{A}=j(A)+d F$ where $A \in H_{2}^{S}(X)$. Recall from Lemma 5.1 that $\tilde{A}$ might contribute to $S\left(\pi_{\ell}\right)$ only if the following three conditions are satisfied:

(1) $d \leq 1$.

(2) $[\Sigma] \cdot A=1-d$.

(3) $\quad A \in \mathcal{R}(X)$ where $\mathcal{R}(X) \subset H_{2}^{S}(X)$ is the positive cone generated by those classes that can be represented by $J_{0}$-holomorphic rational curves. (See Section 5.)

Moreover, $d=1$ iff $A=0$.

The case $d=1$ has already been treated in (18). Assume that $d \leq 0$. A simple computation shows that

$$
\langle[\tilde{\Omega}], \tilde{A}\rangle=2-d, \quad\left\langle c_{1}^{v}, \tilde{A}\right\rangle=-1+\left\langle c_{1}^{X}-h, A\right\rangle .
$$


Here, $h \in H^{2}(X)$ is the hyperplane class corresponding to the embedding $X \subset \mathbb{C} P^{N}$, that is, $h=\mathrm{PD}([\Sigma])$. This proves the following theorem.

Theorem 9.1. Let $X \subset \mathbb{C} P^{N}$ be an algebraic manifold with small dual and $\Sigma \subset X$ a hyperplane section. Then the Seidel element $S\left(\pi_{\ell}\right)$ corresponding to the fibration $\pi_{\ell}$ : $\tilde{X} \longrightarrow \ell$ is given by

$$
S\left(\pi_{\ell}\right)=\left[\omega_{\Sigma}\right] T+\sum_{d \leq 0, A} \mathcal{S}(j(A)+d F) T^{2-d} q^{\left(C_{1}^{X}(A)-h(A)\right) / C_{\Sigma}},
$$

where the sum is taken over all $d \leq 0$ and $A \in \mathcal{R}(X)$ with

(1) $h(A)=1-d$.

(2) $3-d-n \leq c_{1}^{X}(A) \leq 2-d$.

In particular, if $-K_{X}-\Sigma$ is nef and $\min \left\{\left(-K_{X}-\Sigma\right) \cdot A \mid A \in \mathcal{R}(X)\right\} \geq 2$ then

$$
S\left(\pi_{\ell}\right)=\left[\omega_{\Sigma}\right] T
$$

Note that the powers of $T$ in the second summand of (19) are always $\geq 2$ and the powers of $q$ in the second summand are always $\leq 1$ (but might in general be also negative).

Here is a nonmonotone example, not covered by Theorem 4.2 but to which Theorem 9.1 does apply. Let $X=\mathbb{C} P^{m+r} \times \mathbb{C} P^{m}$ with $m \geq 2$ and $r \geq 1$ be embedded in $\mathbb{C} P^{(m+1)(m+r+1)-1}$ by the Segre embedding. It is well known that $\operatorname{def}(X)=r$ (see [46, Theorem 6.5]). It is easy to see that $c_{1}^{X}-h$ is ample and since $m \geq 2$ its minimal value on $\mathcal{R}(X)$ is $m \geq 2$. It follows that $S\left(\pi_{\ell}\right)=\left[\omega_{\Sigma}\right] T \in \mathrm{OH}^{2}(\Sigma ; \mathcal{A})$.

This calculation fails to be true when $m=1$, as will be shown in Section 9.1.

\subsection{A nonmonotone example}

Consider the algebraic manifold $\Sigma=\mathbb{C} P^{1} \times \mathbb{C} P^{1}$. Denote by $f, s \in H_{2}(\Sigma ; \mathbb{Z})$ the classes

$$
f=\left[\mathrm{pt} \times \mathbb{C} P^{1}\right], \quad s=\left[\mathbb{C} P^{1} \times \mathrm{pt}\right] .
$$

We have $H_{2}^{S}(\Sigma)=H_{2}(\Sigma ; \mathbb{Z})=\mathbb{Z} s \oplus \mathbb{Z} f$. Denote by $\alpha, \beta \in H^{2}(\Sigma)$ the Poincaré duals of $f$ and $s$, respectively, that is,

$$
\langle\alpha, s\rangle=1, \quad\langle\alpha, f\rangle=0, \quad\langle\beta, s\rangle=0, \quad\langle\beta, f\rangle=1 .
$$


A simple computation shows that

$$
c_{1}^{\Sigma}=2 \alpha+2 \beta
$$

Before we continue, a small remark about our algebro-geometric conventions is in order. For a complex vector space $V$ we denote by $\mathbb{P}(V)$ the space of complex lines through 0 (not the space of hyperplanes or one-dimensional quotients of $V$ ). Similarly, for a vector bundle $E \rightarrow B$ we denote by $\mathbb{P}(E) \rightarrow B$ the fiber bundle whose fiber over $x \in B$ is $\mathbb{P}\left(E_{X}\right)$, as just defined, that is, the space of lines through 0 in $E_{X}$. We denote by $T \rightarrow \mathbb{P}(E)$ the tautological bundle, which by our convention, is defined as the line bundle whose fiber over $l \in \mathbb{P}\left(E_{X}\right)$ is the line $l$ itself. We denote by $T^{*}$ the dual of $T$, that is, $T_{l}^{*}=\operatorname{hom}\left(l, \mathbb{C}\right.$ ). For example, with these conventions, for $E=\mathbb{C}^{n+1}$ (viewed as a bundle over $B=\mathrm{pt}$ ) we have $T^{*}=\mathcal{O}_{\mathbb{C} P^{n}}(1)$, and $T^{*}$ is ample.

Consider now the bundle $\mathcal{O}_{\mathbb{C} P^{1}}(-1)$ over $\mathbb{C} P^{1}$. There is an obvious inclusion

$$
\iota: \mathcal{O}_{\mathbb{C} P^{1}}(-1) \longrightarrow \mathcal{O}_{\mathbb{C} P^{1}} \oplus \mathcal{O}_{\mathbb{C} P^{1}}
$$

coming from viewing an element $l \in \mathbb{C} P^{1}$ as a subspace $l \subset \mathbb{C} \oplus \mathbb{C}$. Consider now the inclusion:

$$
\mathcal{O}_{\mathbb{C} P^{1}}(-1) \oplus \mathcal{O}_{\mathbb{C} P^{1}}(-1) \stackrel{\mathrm{id} \oplus l}{\longrightarrow} \mathcal{O}_{\mathbb{C} P^{1}}(-1) \oplus \mathcal{O}_{\mathbb{C} P^{1}} \oplus \mathcal{O}_{\mathbb{C} P^{1}}
$$

Denote by $E$ the bundle on the right-hand side of this inclusion and by $E^{\prime}$ the bundle on the left-hand side. Put

$$
X=\mathbb{P}(E)
$$

and denote by pr: $X \longrightarrow \mathbb{C} P^{1}$ the bundle projection. Note that $\mathbb{P}\left(E^{\prime}\right) \cong \mathbb{P}\left(\mathcal{O}_{\mathbb{C} P^{1}} \oplus \mathcal{O}_{\mathbb{C} P^{1}}\right)=$ $\mathbb{C} P^{1} \times \mathbb{C} P^{1}=\Sigma$ hence (22) induces an embedding $i_{\Sigma, X}: \Sigma \longrightarrow X$. Let $T \rightarrow X$ be the tautological bundle (as previously defined) and consider the bundle

$$
\mathcal{L}=T^{*} \otimes p^{*} \mathcal{O}_{\mathbb{C} P^{1}}(1)
$$

Proposition 9.2. The line bundle $\mathcal{L}$ is very ample and the projective embedding of $X$ induced by it has def $=1$. The embedding of $\Sigma, i_{\Sigma, X}(\Sigma) \subset X$, is a smooth hyperplane section of the projective embedding of $X$ induced by $\mathcal{L}$. Moreover if $\omega_{X}$ is the symplectic structure on $X$ induced by the projective embedding of $\mathcal{L}$ and $\omega_{\Sigma}=i_{\Sigma, X}^{*} \omega_{X}$, then we have

$$
\left[\omega_{\Sigma}\right]=2 \alpha+\beta
$$


If $\ell$ is a pencil in the linear system $|\mathcal{L}|$ lying in the complement of the dual variety $X^{*}$, then the Seidel element of the fibration $\pi_{\ell}: \tilde{X} \longrightarrow \ell$ associated to $\ell$ is

$$
S\left(\pi_{\ell}\right)=(2 \alpha+\beta) T+\beta T^{2} .
$$

The details of this calculation are somewhat lengthy and can be found in the expanded version of the paper [14]. One can easily generalize the above example to other projective bundles and also to higher dimensions.

Note that $\left[\omega_{\Sigma}\right]$ and $c_{1}^{\Sigma}$ are not proportional hence the conditions of Theorem 4.2 are not satisfied. It is also easy to see that (for homological reasons) $\left(\Sigma, \omega_{\Sigma}\right)$ does not contain any Lagrangian spheres (cf. Theorems 8.1 and 8.2).

The quantum product for $\left(\Sigma, \omega_{\Sigma}\right)$ is given by (see [37]):

$$
\alpha * \alpha=q T^{2}, \quad \beta * \beta=q T, \quad \alpha * \beta=\alpha \cup \beta \quad \text { where } \operatorname{deg}(q)=4 .
$$

The inverse of $S\left(\pi_{\ell}\right)$ in quantum cohomology is given by

$$
S\left(\pi_{\ell}\right)^{-1}=\frac{1}{q T^{2}(1-T)^{2}}(-2 \alpha+(1+T) \beta) .
$$

Here, we have written $\frac{1}{(1-T)^{2}}$ as an abbreviation for $\left(\sum_{q=0}^{\infty} T^{j}\right)^{2}$.

In contrast to the situation in Proposition 9.2 we can exhibit the same manifold $\Sigma=\mathbb{C} P^{1} \times \mathbb{C} P^{1}$ as a hyperplane section of a different projective manifold $X^{\prime}$ with def $=0$, but with different induced symplectic structure (cf. Theorem 8.4). This goes as follows. Let $X^{\prime} \subset \mathbb{C} P^{5}$ be the image of the degree-2 Veronese embedding of $\mathbb{C} P^{3}$. It is well known that $\operatorname{def}\left(X^{\prime}\right)=0$. A simple computation shows that a smooth hyperplane section of $X^{\prime}$ is isomorphic to $\Sigma=\mathbb{C} P^{1} \times \mathbb{C} P^{1}$. The symplectic form $\omega_{\Sigma}^{\prime}$ on $\Sigma$ induced from the standard symplectic structure of $\mathbb{C} P^{5}$ satisfies

$$
\left[\omega_{\Sigma}^{\prime}\right]=\alpha+\beta
$$

Note that $\left(\Sigma, \omega_{\Sigma}^{\prime}\right)$ has Lagrangian spheres. To see that, note that (e.g., by Moser argument) $\omega_{\Sigma}^{\prime}$ is diffeomorphic to the split form $\omega_{0}=\sigma \oplus \sigma$, where $\sigma$ is the standard Kähler form on $\mathbb{C} P^{1}$. The symplectic manifold $\left(\Sigma, \omega_{0}\right)$ obviously has Lagrangian spheres, for example, $L=\{(z, w) \mid w=\bar{z}\}$, hence so does $\left(\Sigma, \omega_{\Sigma}^{\prime}\right)$. (cf. Theorems 8.1 and 8.2). 
Finally, note that $\left[\omega_{\Sigma}^{\prime}\right]=\alpha+\beta$ is not invertible in the quantum cohomology $\mathrm{QH}(\Sigma ; \Lambda)$. In fact, a simple computation shows that $(\alpha+\beta) *(\alpha-\beta)=0$.

\section{Discussion and Questions}

Here, we briefly discuss further directions of study arising from the results of the paper.

\subsection{Questions on the symplectic topology of manifolds with small dual}

Consider the class of manifolds $\Sigma$ that appear as hyperplane sections of manifolds $X$ with small dual, viewed as symplectic manifolds. Does the group of Hamiltonian diffeomorphisms $\operatorname{Ham}(\Sigma)$ of such manifolds $\Sigma$ have sepecial properties (from the Geomeric, or algebraic viewpoints)? This question seems very much related to the subcriticality of $X \backslash \Sigma$, and results in this direction have been recently obtained by Borman [16] who found a relation between quasi-morphisms on $\operatorname{Ham}(\Sigma)$ and quasi-morphisms on $\operatorname{Ham}(X)$.

The structure of the fundamental group $\pi_{1}(\mathrm{Ham})$ of the group of Hamiltonian diffeomorphisms of a symplectic manifold has been the subject of many studies in symplectic topology. Still, relatively little is known about the structure of these fundamental groups. (e.g., The pool of known examples of symplectic manifolds with nonsimply connected Ham is quite limited.) It would be interesting to ask whether manifolds with small dual and their hyperplane sections exhibit special properties in terms of $\pi_{1}(\mathrm{Ham})$ or more generally in terms of the topology of Ham.

Here are more concrete questions in this direction. Let $X \subset \mathbb{C} P^{N}$ be a manifold with small dual. Denote $k=\operatorname{def}(X)$ and let $\Sigma \subset X$ be a smooth hyperplane section, endowed with the symplectic structure $\omega_{\Sigma}$ induced from $\mathbb{C} P^{N}$. Denote by $\lambda \in \pi_{1}\left(\operatorname{Ham}\left(\Sigma, \omega_{\Sigma}\right)\right.$ the nontrivial element coming from the fibration in Section 4 using the recipe of Section 3.2.1.

(1) What can be said about the minimal Hofer length of the loops in $\operatorname{Ham}\left(\Sigma, \omega_{\Sigma}\right)$ in the homotopy class $\lambda$ ? More generally, what can be said in general about the length spectrum of $\operatorname{Ham}\left(\Sigma, \omega_{\Sigma}\right)$ with respect to the Hofer metric? Preliminary considerations seem to indicate that at least when $b_{2}(X)=1$ the positive part of the norm of $\lambda \in \pi_{1}\left(\operatorname{Ham}\left(\Sigma, \omega_{\Sigma}\right)\right.$ satisfies $v_{+}(\lambda) \leq \frac{1}{\operatorname{dim}_{\mathbb{C}}(\Sigma)+1}$. It would be interesting to verify this, and more importantly to obtain a bound on $v(\lambda)$. (See $[41,42]$ for the definition of these norms on $\pi_{1}$ (Ham) and ways to calculate them.) 
(2) Can the homotopy class $\lambda$ be represented by a Hamiltonian circle action? Several examples of manifolds with small dual indicate that this might be true. In case a Hamiltonian circle action does exist, is it true that it can be deformed into a holomorphic circle action (i.e. an action of $S^{1}$ by biholomorphisms of $\Sigma)$ ?

(3) In which cases is the element $\lambda$ of finite order? Whenever this is the case, does the order of $\lambda$ has any relation to $k=\operatorname{def}(X)$ ?

(4) In case the order of $\lambda$ is infinite, what can be said about the value of the Calabi homomorphism $\widetilde{\mathrm{Cal}}$ on $\lambda$ ? (We view here $\lambda$ as an element of the universal cover $\widetilde{\operatorname{Ham}}\left(\Sigma, \omega_{\Sigma}\right)$.) See [25] for the definition of $\widetilde{\mathrm{Cal}}$ etc.

Of course, one could ask the same questions also about $X$ itself (rather than $\Sigma$ ). It is currently not known what are the precise conditions insuring that an algebraic manifold $X$ with small dual can be realized as a hyperplane section in an algebraic manifold $Y$ (of one-dimension higher).

Another question, lying at the border between symplectic topology and algebraic geometry is the following. The main results of this paper show that an algebraic manifold $X \subset \mathbb{C} P^{N}$ with small dual and $b_{2}(X)=1$ gives rise to a distinguished nontrivial element $\lambda \in \pi_{1}(\operatorname{Ham}(\Sigma))$ where $\Sigma$ is a hyperplane section of $X$. On the other hand, every homotopy class of loops $\gamma \in \pi_{1}(\operatorname{Ham}(\Sigma))$ gives rise to a Hamiltonian fibration $\pi_{\gamma}: \tilde{M}_{\gamma} \longrightarrow S^{2}$ with fiber $\Sigma$. Consider now (positive as well as negative) iterates $\gamma=\lambda^{r}, r \in \mathbb{Z}$, of $\lambda$ and the Hamiltonian fibrations corresponding to them $\pi_{\lambda^{r}}: \tilde{M}_{\lambda^{r}} \longrightarrow S^{2}$. Do these fibrations correspond to an embedding of $\Sigma$ as a hyperplane section in some algebraic manifold with positive defect? Or more generally, do the fibrations $\pi_{\lambda^{r}}$ correspond to some geometric framework involving the algebraic geometry of $\Sigma$ and its projective embeddings? It seems tempting to suspect that $\lambda^{2}$, for example, corresponds to a fibration similar to $\pi_{\ell}: \tilde{X} \longrightarrow \ell \approx S^{2}$ (see Section 4) but instead of taking $\ell$ to be a line in the complement of $X^{*}$ one takes $\ell$ to be a degree 2 curve in the complement of $X^{*}$.

Finally, here is another general question motivated by analogies to algebraic geometry. Can the concept of manifolds with small dual be generalized to symplectic manifolds? Can one define a meaningful concept of defect? The motivation comes from the following framework. Let $(X, \omega)$ be a closed integral symplectic manifold (integral means that $[\omega]$ admits a lift to $H^{2}(X ; \mathbb{Z})$ ). By a theorem of Donaldson [18] $X$ admits symplectic hyperplane sections, that is, for $k \gg 0$, there exists a symplectic submanifold $\Sigma$ representing the Poincaré dual to $k[\omega]$. (Moreover, the symplectic generalization of the notion of Lefschetz pencil, exists too [19].) Suppose now that for some such $\Sigma$ the manifold $X \backslash \Sigma$ is subcritical. Does this imply on $\Sigma$ and $X$ results similar to what we 
have obtained in this paper? (e.g., is $\left[\left.\omega\right|_{\Sigma}\right]$ invertible in $\mathrm{OH}(\Sigma)$ ?) One of the difficulty in this type of questions is that the concept of dual variety (of a projective embedding, or of a linear system) does not exist in the realm of symplectic manifolds and their Donaldson hyperplane sections. Note that we are not aware of examples of pairs $(X, \Sigma)$ with $X \backslash \Sigma$ subcritical that are not equivalent (e.g., symplectomorphic) to algebraic pairs $\left(X^{\prime}, \Sigma^{\prime}\right)$.

\subsection{Ouestions about the algebraic geometry of manifolds with small dual}

We have seen that for hyperplane sections $\Sigma$ of manifolds with small dual $X \subset \mathbb{C} P^{N}$, $\left[\omega_{\Sigma}\right] \in \mathrm{OH}^{2}(\Sigma ; \Lambda)$ is invertible. Is the same true for $X$, that is, is $\left[\omega_{X}\right] \in \mathrm{OH}^{2}(X ; \Lambda)$ an invertible element? The 2-periodicity of the Betti number of $X$ in Corollary B indicates that this might be the case. Note that our proof of the 2-periodicity for $X$ was based on the 2-periodicity for $\Sigma$ (which in turn comes from the invertibility of $\left[\omega_{\Sigma}\right]$ ), together with some Lefschetz-type theorems, and did not involve any quantum cohomology considerations for $X$.

Another circle of questions has to do with Theorem 9.1. It would be interesting to figure out more explicitly the terms with $d \leq 0$ in formula (19). This might be possible to some extent of explicitness using Mori theory in the special case of manifolds with small dual (see, e.g., [4, 5, 47] and the references therein). In the same spirit, it would be interesting to see if there are any topological restrictions on $\Sigma$ and $X$ coming from the invertibility of $S\left(\pi_{\ell}\right)$ in the nonmonotone case. We remark that when $\left(\Sigma, \omega_{\Sigma}\right)$ is not spherically monotone one should work with a more complicated Novikov ring $\mathcal{A}$ as explained in Section 9.

Another interesting question has to do with the structure of the quantum cohomology $\mathrm{OH}^{*}(\Sigma ; \Lambda)$ of hyperplane sections $\Sigma$ of manifolds with small dual $X$. As a corollary of Theorem A we have obtained that in the monotone case $\operatorname{OH}^{*}(\Sigma ; \Lambda)$ satisfies the relation $\left[\omega_{\Sigma}\right] * \alpha=q$ for some $\alpha \in H^{n+k-2}(\Sigma)$. In some examples this turns out to be the only relation. Thus, it is tempting to ask when do we have a ring isomorphism

$$
\mathrm{QH}^{*}(\Sigma ; \Lambda) \cong \frac{\left(H^{\bullet}(\Sigma) \otimes \Lambda\right)^{*}}{\langle\omega * \alpha=q\rangle}
$$

In a similar context, it is interesting to note that the algebraic structure of quantum cohomology of uniruled manifolds has been studied in a recent paper of McDuff [35]. In particular, in [35] McDuff proves a general existence result for nontrivial invertible elements of the quantum cohomology of uniruled manifolds using purely algebraic 
methods. One can view part of the results in this paper as a direct computation in the case of manifolds with positive defect.

\section{Acknowledgements}

We would like to thank the referee for pointing out to us the reference to the (second) paper of Andreotti and Frankel [2] and for useful remarks helping to improve the quality of the exposition.

\section{References}

[1] Andreotti, A. and T. Frankel. "The Lefschetz theorem on hyperplane sections." Annals of Mathematics. Second Series 69 (1959): 713-7.

[2] Andreotti, A. and T. Frankel. "The Second Lefschetz Theorem on Hyperplane Sections." In Global Analysis (Papers in Honor of K. Kodaira), 1-20. Tokyo: University Tokyo Press, 1969.

[3] Arnold, V. "Some Remarks on Symplectic Monodromy of Milnor Fibrations." In The Floer Memorial Volume, 99-103. Progress in Mathematics 133. Basel: Birkhäuser, 1995.

[4] Beltrametti, M., M. Fania, and A. Sommese. "On the discriminant variety of a projective manifold." Forum Mathematicum 4, no. 6 (1992): 529-47.

[5] Beltrametti, M. and A. Sommese. The Adjunction Theory of Complex Projective Varieties. de Gruyter Expositions in Mathematics 16. Berlin: Walter de Gruyter \& Co., 1995.

[6] Biran, P. "Geometry of Symplectic Intersections." In Proceedings of the International Congress of Mathematicians, vol. II, 241-55. Beijing: Higher Ed. Press, 2002.

[7] Biran, P. "Symplectic Topology and Algebraic Families." In Proceedings of the 4'th European Congress of Mathematics, Stockholm (2004), 827-36. Zürich: European Mathematical Society, 2005.

[8] Biran, P. "Lagrangian non-intersections." Geometric and Functional Analysis 16, no. 2 (2006): 279-326.

[9] Biran, P. and K. Cieliebak. "Symplectic topology on subcritical manifolds." Commentarii Mathematici Helvetici, 76, no. 4 (2002): 712-53.

[10] Biran, P. and K. Cieliebak. "Lagrangian embeddings into subcritical Stein manifolds." Israel Journal of Mathematics, 127 (2002): 221-44.

[11] Biran, P. and O. Cornea. "Quantum structures for Lagrangian submanifolds." (2007): preprint. Can be found at http://arxiv.org/pdf/0708.4221.

[12] Biran, P. and O. Cornea. "A Lagrangian Quantum Homology." In New Perspectives and Challenges in Symplectic Field Theory, 1-44. CRM Proceedings \& Lecture Notes 49. Providence, RI: American Mathematical Society, 2009.

[13] Biran, P. and O. Cornea. "Rigidity and uniruling for Lagrangian submanifolds." Geometry and Topology 13, no. 5 (2009): 2881-989.

[14] Biran, P. and Y. Jerby. "The symplectic topology of projective manifolds with small dual." Expanded version, 2012. Can be found at http://arxiv.org/pdf/1107.0174v2. 
[15] Biran, P. and M. Khanevsky. "A floer-gysin exact sequence for Lagrangian submanifolds." (2011): preprint. Can be found at http://arxiv.org/pdf/1101.0946.

[16] Borman, M. S. "Symplectic reduction of quasi-morphisms and quasi-states." (2010): preprint, can be found at http://arxiv.org/pdf/1007.4036.

[17] Donaldson, S. "Polynomials, Vanishing Cycles and Floer Homology." In Mathematics: Frontiers and Perspectives, 55-64. Providence, RI: American Mathematical Society, 2000.

[18] Donaldson, S. K. "Symplectic submanifolds and almost-complex geometry." Journal of Differential Geometry 44, no. 4 (1996): 666-705.

[19] Donaldson, S. K. "Lefschetz pencils on symplectic manifolds." Journal of Differential Geometry 53, no. 2 (1999): 205-36.

[20] Ein, L. "Varieties with small dual varieties. II." Duke Mathematical Journal 52, no. 4 (1985): 895-907.

[21] Ein, L. "Varieties with small dual varieties. I." Inventiones Mathematicae 86, no. 1 (1986): 63-74.

[22] Eliashberg, Y. "Topological characterization of Stein manifolds of dimension > 2." International Journal of Mathematics 1, no. 1 (1990): 29-46.

[23] Eliashberg, Y. "Symplectic Geometry of Plurisubharmonic Functions." In Gauge Theory and Symplectic Geometry, 49-67. NATO Advanced Science Institutes Series C: Mathematical and Physical Sciences 488. Dordrecht: Kluwer Academic Publisher, 1997.

[24] Eliashberg, Y. and M. Gromov. "Convex Symplectic Manifolds." In Several Complex Variables and Complex Geometry Part 2 (Santa Cruz, CA, 1989), 135-62. Proceedings of Symposia in Pure Mathematics 52. Providence, RI: American Mathematical Society, 1991.

[25] Entov, M. and L. Polterovich. "Calabi quasimorphism and quantum homology." International Mathematics Research Notices no. 30 (2003): 1635-76.

[26] Griffiths, P. and J. Harris. Principles of Algebraic Geometry. Pure and Applied Mathematics. New York: John Wiley \& Sons, Inc., 1978.

[27] Griffiths, P. and J. Harris. "Algebraic geometry and local differential geometry." Annales Scientifiques de l'École Normale Supérieure. Quatriéme Série 12, no. 3 (1979): 355-452.

[28] Guillemin, V., E. Lerman, and S. Sternberg. Symplectic Fibrations and Multiplicity Diagrams. Cambridge: Cambridge University Press, 1996.

[29] He, J. "Correlators and descendants of subcritical Stein manifolds." (2008): preprint, can be found at http://arxiv.org/pdf/0810.4174.

[30] Kleiman, S. "About the Conormal Scheme." In Complete Intersections (Acireale, 1983), 16197. Lecture Notes in Mathematics 1092. Berlin: Springer, 1984.

[31] Kleiman, S. "Tangency and Duality." In Proceedings of the 1984 Vancouver Conference in Algebraic Geometry, 163-225. CMS Conference Proceedings 6. Providence, RI: American Mathematical Society, 1986.

[32] Landsberg, J. Tensors: Geometry and Applications. Graduate Studies in Mathematics 128. Providence, RI: American Mathematical Society, 2012.

[33] Lanteri, A. and D. Struppa. "Projective manifolds whose topology is strongly reflected in their hyperplane sections." Geometriae Dedicata 21, no. 3 (1986): 357-74. 
[34] Lazarsfeld, R. and A. Van de Ven. Topics in the Geometry of Projective Space. DMV Seminar 4. Basel: Birkhäuser, 1984. Recent work of F. L. Zak, With an addendum by Zak.

[35] McDuff, D. "Hamiltonian $S^{1}$-manifolds are uniruled." Duke Mathematical Journal 146, no. 3 (2009): 449-507.

[36] McDuff, D. and D. Salamon. Introduction to Symplectic Topology, 2nd ed. Oxford Mathematical Monographs. New York: The Clarendon Press, Oxford University Press, 1998.

[37] McDuff, D. and D. Salamon. J-Holomorphic Curves and Symplectic Topology. American Mathematical Society Colloquium Publications 52. Providence, RI: American Mathematical Society, 2004.

[38] Mumford, D. "Some Footnotes to the Work of C. P. Ramanujam." In C. P. Ramanujam-A Tribute, 247-62. Tata Institute of Fundamental Research Studies in Mathematics 8. Berlin: Springer, 1978.

[39] Oh, Y.-G. "Floer cohomology of Lagrangian intersections and pseudo-holomorphic disks. I." Communications on Pure and Applied Mathematics 46, no. 7 (1993): 949-93.

[40] Oh, Y.-G. "Floer cohomology, spectral sequences, and the Maslov class of Lagrangian embeddings." International Mathematics Research Notices 1996, no. 7 (1996): 305-46.

[41] Polterovich, L. "Gromov's $K$-area and symplectic rigidity." Geometric and Functional Analysis 6, no. 4 (1996): 726-39.

[42] Polterovich, L. The Geometry of the Group of Symplectic diffeomorphisms. Lectures in Mathematics ETH Zürich. Basel: Birkhäuser, 2001.

[43] Seidel, P. "Floer homology and the symplectic isotopy problem." PhD thesis, Oxford University, 1997.

[44] Seidel, P. " $\pi_{1}$ of symplectic automorphism groups and invertibles in quantum homology rings." Geometric and Functional Analysis 7, no. 6 (1997): 1046-95.

[45] Snow, D. "The nef value and defect of homogeneous line bundles." Transactions of the American Mathematical Society 340, no. 1 (1993): 227-41.

[46] Tevelev, E. "Projectively dual varieties." Manuscript (2001). Can be found at http://arxiv.org/pdf/math/0112028.

[47] Tevelev, E. "Projectively dual varieties." Journal of Mathematical Sciences 117, no. 6 (2003): 4585-732.

[48] Zak, F. Tangents and Secants of Algebraic Varieties. Translations of Mathematical Monographs 127. Providence, RI: American Mathematical Society, 1993. 